\title{
On the Performance of Hadamard Ratio
}

\section{Detector-based Spectrum Sensing for Cognitive Radios}

\author{
Saeid Sedighi, Abbas Taherpour, Member, IEEE, Josep Sala, Senior Member, \\ IEEE and Tamer Khattab, Member, IEEE
}

\begin{abstract}
We consider the problem of multiantenna spectrum sensing in Cognitive Radios (CRs) when the receivers are assumed to be uncalibrated across the antennas. The performance of the Hadamard Ratio Detector (HRD) is analyzed in such a scenario. Specifically, we first derive the exact distribution of the HRD statistic under the null hypothesis, which leads to an elaborate but closed-form expression for the false-alarm probability. Then, we derive a simpler and tight closed-form approximation for both the false-alarm and detection probabilities by using a moment-based approximation of the HRD statistical distribution under both hypotheses. Finally, the accuracy of the obtained results is verified by simulations.
\end{abstract}

\section{Index Terms}

Cognitive radio, spectrum sensing, multiple antennas, Generalized Likelihood Ratio Test (GLRT), uncalibrated receivers, performance evaluation.

Copyright (c) 2015 IEEE. Personal use of this material is permitted. However, permission to use this material for any other purposes must be obtained from the IEEE by sending a request to pubs-permissions@ieee.org.

The research work of Saeid Sedighi, Abbbas Taherpour and Tamer Khattab is supported by a grant from Qatar National Research Fund (a member of Qatar Foundation) under grant number NPRP 6-1326-2-532. The statements made therein are the sole responsibility of the authors. The work of Josep Sala is supported by projects: TEC2010-21245-C02/TCM (DYNACS), TEC2013-47020-C2-2-R (COMPASS) (Ministerio de Economia y Competitividad, Spanish National Research Plan) and 2014-SGR-60 AGAUR (Catalan Government).

Saeid Sedighi and Abbas Taherpour are with the Dept. of Electrical Engineering, Imam Khomeini International University (IKIU), Qazvin, Iran (e-mails: \{s.sedighi, taherpour\}@eng.ikiu.ac.ir).

Josep Sala is with the Dept. of Signal Theory and Communications, Universitat Politècnica de Catalunya - Barcelona Tech (UPC), Barcelona, Spain (e-mail: josep.sala@upc.edu).

Tamer Khattab is with the Dept. of Electrical Engineering, Qatar University, Doha, Qatar (e-mail: tkhattab@ieee.org). 


\section{INTRODUCTION}

The identification of spectrum holes by Secondary Users (SUs) constitutes a major requirement at the physical layer of Cognitive Radio (CR) networks, where Spectrum Sensing (SS) techniques are sought to attain a sufficiently reliable detection probability over the shortest possible sensing time. So far, different methods have been proposed for SS [1], [2]. The Energy Detector (ED) is such a popular method to detect an unknown signal in additive white noise [3], [4]. However, it requires knowledge of the noise variance to set the decision threshold, which, in practice, has to be estimated under errors introduced by the detection device and the environment, e.g., temperature, humidity, device aging, radio interference, etc.. It has been shown that to achieve a desired probability of detection under such unavoidable noise variance uncertainties, the Signalto-Noise Ratio (SNR) must be above a certain threshold [5] (SNR wall).

An efficient strategy to increase the reliability of SS is to use cooperative sensing, in which information from multiple spatially distributed SUs is incorporated for detecting the Primary User (PU) [6], as recently addressed in [7]-[11]. [7] studies optimization of Cooperative SS (CSS) with an improved ED in each SU over imperfect reporting channels. CSS for a CR mesh network is considered in [8]. A linear cooperative sensing framework based on the combination of the observed energies by different SUs is proposed in [9]. In [10], [11], the authors propose a selective-relay based CSS scheme without a dedicated reporting channel, which is able to control and reduce the interference from SUs to the PU.

Using multiple antennas at the SU receiver is a possible approach to tackle noise uncertainty, to improve the performance of SS by exploiting available observations in the spatial domain and also to avoid a (probably imperfect) reporting channel like that needed for CSS. Nevertheless, multiantenna SS does present some disadvantages with respect to CSS: i.e., its inability to counteract the hidden node problem as well as a comparatively more expensive implementation. Multiantenna techniques have been addressed in [12]-[21]. [12] a blind SS approach is adopted in which the empirical characteristic function of the multiantenna samples is used to formulate the statistical test. In [13], the authors derive the optimum Neyman-Pearson (NP) and sub-optimum Generalized Likelihood Ratio Test (GLRT)-based multiantenna detectors of an Orthogonal Frequency Division Multiplexing (OFDM) signal with a cyclic prefix of known length. The Rao test is applied to derive sub-optimum multiantenna detectors under the correlated 
receiving antennas model in [14]. Multiantenna SS in frequency selective channels is addressed in [15]. In [16]-[19], the authors derive the GLRT detectors of spatial rank-one PU signals robust to noise variance uncertainty. Finally, some GLRT eigenvalue-based detectors for multiantenna SS are proposed in [20], [21] for PU signals with spatial rank larger than one.

Although all detectors proposed in [12]-[21] are robust to noise uncertainty, their performance is sensitive to nonuniform per-antenna noise variances as may occur due to calibration errors. To overcome this drawback, various GLRT-based detectors for uncalibrated multiantenna receivers have been proposed in [22], [23], showing that the corresponding GLRT detector for an unstructured signal covariance matrix is given by the Hadamard ratio of the sample covariance matrix. But neither [22] nor [23] have completely investigated the performance of the Hadamard Ratio Detector (HRD): although both papers offer a useful asymptotic expression for its false-alarm probability, those, are not accurate for small sample sizes. Moreover, no analytical expression for the detection probability of the HRD has been derived in [22], [23], which is evaluated only by Monte Carlo simulations. A number of approximations to the distribution of the HRD have been reported: in [24], a moment-based beta approximation to the HRD distribution under the null hypothesis is given, which, although approximately accurate, is not amenable to a physical and meaningful intuitive interpretation; in [25] the authors adopt the asymptotic chi-squared distribution of the GLRT for both the false-alarm and detection probabilities of the HRD which is accurate only for a large sample size.

In this work, we analyze in detail the detection and false-alarm probabilities of the HRD. We first derive the exact distribution of the HRD statistic under the null hypothesis by using the Mellin transform, which leads to an analytical expression for the complex moments of the test and its false-alarm probability. Nevertheless, the exact analytical expression involves Meijers Gfunction whose numerical evaluation is computationally demanding and its interpretation difficult. Thus, alternatively, we derive a simple and tight analytical approximation to the false-alarm probability by using a moment-based approximation to the HRD statistical distribution under the null hypothesis, with affordable computational complexity for the accurate determination of the decision threshold. We also provide an intuitive interpretation for the impact of different parameters on the proposed approximation. Additionally, we obtain an expression for all integer moments of the test under $\mathcal{H}_{1}$ and a simple tight analytical approximation to the detection probability by using a moment-based approximation to the HRD statistical distribution, which 
is accurate at all sample sizes. Simulation results verify the obtained exact analytical expression for the false-alarm probability and show that the proposed analytical approximations: (i) closely match Monte Carlo simulations for the false-alarm and detection probabilities; (ii) the expression for the false-alarm probability is more accurate than the asymptotic analytical expression proposed in [22] and [23] for all values of the sample size.

The rest of the paper is organized as follows: in Section II, we describe the system model and present the HRD. In Section III, we derive the exact and approximate closed-form expressions for the false-alarm probability. The closed-form approximation to the detection probability is obtained in Section IV. Simulations and related discussions are given in Section V. Finally, conclusions are drawn in Section VI.

Notation: lightface denotes scalars. Vectors and matrices are referred to by lower- and uppercase boldface, respectively. $a_{i, j}$ and $c_{i}$ stand for the entries of matrix $\mathbf{A}$ and vector $\mathbf{c}$, resp.. $\mathbb{C}$ and $\mathbb{N}$ denote the sets of all complex and natural numbers, resp.. The superscripts $T$ and $H$ denote the transpose and Hermitian (conjugate transpose) operations, resp.. $j$ is the imaginary unit, i.e., $j^{2}=-1$. The $M \times M$ identity matrix is $\mathbf{I}_{M} \cdot \operatorname{diag}\{\mathbf{A}\}$ and $\operatorname{diag}\left\{c_{1}, c_{2}, \cdots, c_{M}\right\}$ are diagonal matrices with diagonal entries equal to those of $\mathbf{A}$ and $c_{1}, c_{2}, \cdots, c_{M}$, resp.. The real and imaginary parts of $c$ are $\Re\{c\}$ and $\Im\{c\}$, resp.. E $\{\cdot\}$ is the statistical expectation. $\left(c_{1}, c_{2}, \cdots, c_{d}\right)$ denote a $d$-tuple. $\operatorname{tr}(\mathbf{A}), \operatorname{etr}(\mathbf{A}),|\mathbf{A}|$ and $\|\mathbf{A}\|_{F}^{2}$ denote the trace, $\exp (\operatorname{tr}(\mathbf{A}))$, determinant and Frobenius norm of the matrix $\mathbf{A}$, resp.. $\mathbf{A} \succ \mathbf{0}$ indicates that matrix $\mathbf{A}$ is positive definite. $\mathcal{C N}(\mathbf{c}, \mathbf{P})$ denotes the circular complex Gaussian distribution with mean $\mathbf{c}$ and covariance matrix P. $\mathcal{W}_{C}(M, L, \mathbf{P})$ denotes the complex Wishart distribution of dimension $M$ and $L$ degrees of freedom, with covariance matrix P. $O(\cdot)$ and $o(\cdot)$ denote Landau's big and small-o, resp..

\section{Problem Formulation}

\section{A. System Model}

We assume a SU node fitted with $M$ antennas that senses a given frequency band simultaneously accessed by the $K$ different PUs. Let $\mathcal{H}_{1}$ and $\mathcal{H}_{0}$ denote, respectively, the hypotheses of the presence and absence of PUs. The hypothesis testing problem for such a scenario can be 
formulated as

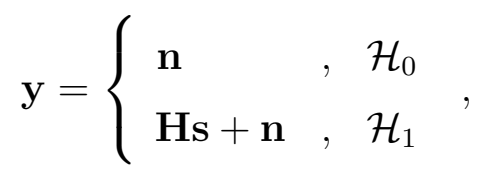

with $\mathbf{y} \in \mathbb{C}^{M}$ the received signal vector, $\mathbf{H} \in \mathbb{C}^{M \times K}$ the unknown channel gains matrix between the $K$ PUs and the $M$ receiving antennas, $\mathbf{s} \in \mathbb{C}^{K}$ the transmitted signals from the PUs and $\mathbf{n} \in \mathbb{C}^{M}$ the additive noise vector, assumed zero-mean circular complex Gaussian distributed with unknown diagonal covariance matrix $\Sigma=\operatorname{diag}\left\{\sigma_{1}^{2}, \sigma_{2}^{2}, \ldots, \sigma_{\mathrm{M}}^{2}\right\}$. The per-antenna noise variances $\sigma_{i}^{2}$ are assumed different to reflect potential tolerances in the components of different RF chains. For the theoretical development, we model s as a zero-mean circular complex Gaussian random vector. We adopt a Gaussian model for the PU signal for the following reasons. Firstly, [26][28] conclude that OFDM signals are the best physical layer candidate for CR, which is thus expected of most practical CR systems. In that, a Gaussian model is accurate for OFDM signals with a sufficiently large number of subcarriers. Secondly, under asynchronous sampling, the actual distribution of the PU signal is unknown; since the noise is assumed Gaussian as well, the Gaussian Probability Density Function (PDF) for the PU is the least informative for the detection problem and also widely used by other researchers [18], [21], [32]-[34]. Thirdly, the Gaussian model is tractable and yields useful schemes whose results are also approximately accurate for other distributions [14] as attested by simulations in section $\mathrm{V}$ for scenarios featuring modulated PU signals with actual non-Gaussian distribution.

Without loss of generality, we assume that $\mathbf{s}$ has an identity covariance matrix as any correlation and scaling of the PUs signals can be incorporated into the channel gains matrix $\mathbf{H}$. Under $\mathcal{H}_{1}$, the PUs signals, i.e., $\mathbf{s}$ and noise vector, i.e., $\mathbf{n}$ are assumed mutually independent. In consequence, the received signal vector $\mathbf{y}$ has a zero-mean circular complex Gaussian distribution, i.e., $\mathbf{y} \sim$ $\mathcal{C N}(\mathbf{0}, \mathbf{Q})$, where

$$
\begin{cases}\mathcal{H}_{0} & : \mathbf{Q}=\mathbf{\Sigma} \\ \mathcal{H}_{1} & : \quad \mathbf{Q}=\mathbf{H H}^{H}+\mathbf{\Sigma}\end{cases}
$$

\section{B. Hadamard Ratio Detector}

Let $\mathbf{Y}=\left[\mathbf{y}_{1}, \cdots, \mathbf{y}_{L}\right] \in \mathbb{C}^{M \times L}$ be a complex matrix containing $L$ i.i.d snapshots $\mathbf{y}_{1}, \cdots, \mathbf{y}_{L}$ from model (1). We assume a slow-fading channel in which the channel gains matrix $\mathbf{H}$ remains 
constant during the sensing time. Let $\mathbf{R}$ denote the sample covariance defined as

$$
\mathbf{R}=\mathbf{Y} \mathbf{Y}^{H}
$$

In practice, the $\mathrm{SU}$ is ignorant of the number of active PUs, i.e., $K$. Hence, no specific structure can be assumed for the covariance matrix $\mathbf{Q}$ under $\mathcal{H}_{1}$ except that it is positive definite. The corresponding GLRT for such a scenario has been formulated in [22], [23], and is given by the Hadamard ratio of the sample covariance matrix

$$
T=\frac{\prod_{i=1}^{M} r_{i, i}}{|\mathbf{R}|} \underset{\mathcal{H}_{1}}{\gtrless} \mathcal{H}_{0} \eta,
$$

with $\eta$ the decision threshold for a given false-alarm probability. As noted (Introduction), no accurate analytical expressions for the false-alarm and detection probabilities of the HRD are available yet. Thus, we address the detailed analytical performance study of the HRD in the forthcoming sections.

\section{FAlse-Alarm Probability}

In this section, we derive the exact closed-form expression for the false-alarm probability of the HRD by using the Mellin transform, as well as a simpler and closed-form approximation to the false-alarm probability of the HRD by using a moment-based approximation of the distribution of $T$.

\section{A. Exact Expression}

For a random variable $X$, the Mellin Transform of its PDF is expressed as its $(z-1)$-th complex moment: $\mathcal{M}_{X}(z) \doteq \mathbb{E}\left[X^{z-1}\right]$. Conversely, $f_{X}(x)$ may be recovered with the inverse Mellin Transform,

$$
f_{X}(x)=\frac{1}{2 \pi \mathrm{j}} \int_{c-\mathrm{j} \infty}^{c+\mathrm{j} \infty} \mathcal{M}_{X}(z) x^{-z} \mathrm{~d} z
$$

Let us proceed to evaluate the complex moments of the decision statistic $\Lambda$,

$$
\Lambda \doteq \frac{1}{T}=\frac{|\mathbf{R}|}{\prod_{i=1}^{M} r_{i, i}} \underset{\mathcal{H}_{1}}{\gtrless \mathcal{H}_{0}} \eta,
$$

Lemma 1. Under $\mathcal{H}_{0}$, the random variable $\prod_{i=1}^{M} r_{i, i}$ is independent of the decision statistic $T$.

Proof: See appendix A. 
From Lemma 1 and $z \in \mathbb{C}$, we have,

$$
\mathrm{E}\left\{|\mathbf{R}|^{z}\right\}=\mathrm{E}\left\{\left(\Lambda \prod_{i=1}^{M} r_{i, i}\right)^{z}\right\}=\mathrm{E}\left\{\Lambda^{z}\right\} \mathrm{E}\left\{\left(\prod_{i=1}^{M} r_{i, i}\right)^{z}\right\},
$$

from which

$$
\mathrm{E}\left\{\Lambda^{z}\right\}=\frac{\mathrm{E}\left\{|\mathbf{R}|^{z}\right\}}{\mathrm{E}\left\{\left(\prod_{i=1}^{M} r_{i, i}\right)^{z}\right\}},
$$

Let us consider the denominator of (8): from [35, Theorems 3.2, 3.5 and 3.6], under $\mathcal{H}_{0}, r_{i, i}$ is distributed as $r_{i, i} \sim \frac{1}{2} \sigma_{i}^{2} \chi_{2 L}^{2}$, with $\chi_{2 L}^{2}$ a chi-squared random variable with $2 L$ degrees of freedom and where the random variables $\left\{r_{i, i}\right\}_{i=1}^{M}$ are mutually independent for different $i$ 's. Thus,

$$
\begin{aligned}
\mathrm{E}\left\{\left(\prod_{i=1}^{M} r_{i, i}\right)^{z}\right\} & =\prod_{i=1}^{M} \frac{\sigma_{i}^{2 z}}{2^{z}} \int_{0}^{\infty} r_{i, i}^{z} \frac{r_{i, i}^{L-1} e^{-r_{i, i} / 2}}{2^{L} \Gamma(L)} \mathrm{d} r_{i, i} \\
& =\prod_{i=1}^{M} \frac{\sigma_{i}^{2 z}}{2^{L+z} \Gamma(L)} \int_{0}^{\infty} r_{i, i}^{L+z-1} e^{-r_{i, i} / 2} \mathrm{~d} r_{i, i}
\end{aligned}
$$

Using the complete Gamma function, $\Gamma(z) \doteq \int_{0}^{\infty} t^{z-1} e^{-t} \mathrm{~d} t$,

$$
\mathrm{E}\left\{\left(\prod_{i=1}^{M} r_{i, i}\right)^{z}\right\}=\prod_{i=1}^{M} \frac{\sigma_{i}^{2 z} \Gamma(L+z)}{\Gamma(L)}=\left(\frac{\Gamma(L+z)}{\Gamma(L)}\right)^{M}|\Sigma|^{z} .
$$

Let us now consider the numerator of (8): by [35, Theorem 3.8], we have the following stochastic product decomposition: $|\mathbf{R}| \sim|\Sigma| \prod_{i=1}^{M} V_{i}$, with mutually independent $V_{i} \sim \frac{1}{2} \chi_{2(L-M+i)}^{2}$. Thus,

$$
\begin{aligned}
\mathrm{E}\left\{|\mathbf{R}|^{z}\right\} & =|\Sigma|^{z} \prod_{i=1}^{M} \frac{1}{2^{z}} \int_{0}^{\infty} V_{i}^{z} \frac{V_{i}^{L-M+i-1} e^{-V_{i} / 2}}{2^{L-M+i} \Gamma(L-M+i)} \mathrm{d} V_{i} \\
& =|\Sigma|^{z} \prod_{i=1}^{M} \frac{\int_{0}^{\infty} V_{i}^{L-M+z+i-1} e^{-V_{i} / 2} \mathrm{~d} V_{i}}{2^{L-M+z+i} \Gamma(L-M+i)},
\end{aligned}
$$

which, by using again the complete Gamma function, yields

$$
\mathrm{E}\left\{|\mathbf{R}|^{z}\right\}=|\Sigma|^{z} \prod_{i=1}^{M} \frac{\Gamma(L-M+z+i)}{\Gamma(L-M+i)} .
$$

Let us now construct $\mathrm{E}\left\{\Lambda^{z}\right\}$ in (8): by replacing (10) and (12) in (8), we get,

$$
\mathrm{E}\left\{\Lambda^{z}\right\}=\frac{(\Gamma(L))^{M}}{(\Gamma(L+z))^{M}} \cdot \frac{\prod_{i=1}^{M} \Gamma(L-M+z+i)}{\prod_{i=1}^{M} \Gamma(L-M+i)} .
$$

Note, for cross-checking, that [24, Eq. (20)] provides the integer moments $E\left[\left(T^{(\text {ind })}\right)^{n}\right]$, with $T^{\text {(ind) }}=\Lambda$, by expressing $\Lambda$ as a product of independent beta-distributed random variables. 
Applying the inverse Mellin transform in (5) to $\mathcal{M}_{\Lambda}(z)=\mathrm{E}\left\{\Lambda^{z-1}\right\}$ in (13), we get,

$$
\begin{aligned}
f_{\Lambda \mid \mathcal{H}_{0}}\left(\lambda \mid \mathcal{H}_{0}\right) & =\frac{1}{2 \pi \mathrm{j}} \int_{c-\mathrm{j} \infty}^{c+\mathrm{j} \infty} \frac{(\Gamma(L))^{M}}{(\Gamma(L+z-1))^{M}} \cdot \frac{\prod_{i=1}^{M} \Gamma(L-M+z-1+i)}{\prod_{i=1}^{M} \Gamma(L-M+i)} \cdot \lambda^{-z} \mathrm{~d} z \\
& =\frac{(\Gamma(L))^{M}}{\prod_{i=1}^{M} \Gamma(L-M+i)} \cdot \frac{1}{2 \pi \mathrm{j}} \int_{c-\mathrm{j} \infty}^{c+\mathrm{j} \infty} \frac{\prod_{i=1}^{M} \Gamma(L-M+z-1+i)}{\prod_{i=1}^{M} \Gamma(L+z-1)} \cdot \lambda^{-z} \mathrm{~d} z
\end{aligned}
$$

Simplifying the fraction within the integral in (14), we get,

$$
f_{\Lambda \mid \mathcal{H}_{0}}\left(\lambda \mid \mathcal{H}_{0}\right)=\frac{(\Gamma(L))^{M}}{\prod_{i=1}^{M} \Gamma(L-M+i)} \cdot \frac{1}{2 \pi \mathrm{j}} \int_{c-\mathrm{j} \infty}^{c+\mathrm{j} \infty} \frac{\prod_{i=1}^{M-1} \Gamma(L-M+z-1+i)}{\prod_{i=1}^{M-1} \Gamma(L+z-1)} \cdot \lambda^{-z} \mathrm{~d} z .
$$

By using the definition of Meijer's G-function [36, Sec 9.3, p. 1032] the exact PDF of $\Lambda$ under $\mathcal{H}_{0}$ is obtained as

$$
f_{\Lambda \mid \mathcal{H}_{0}}\left(\lambda \mid \mathcal{H}_{0}\right)=\frac{(\Gamma(L))^{M}}{\prod_{i=1}^{M} \Gamma(L-M+i)} G_{M-1, M-1}^{M-1,0}\left(\begin{array}{c}
a_{1}, \ldots, a_{M-1} \\
b_{1}, \ldots, b_{M-1}
\end{array} \mid \lambda\right)
$$

where $a_{i}=L-1$ and $b_{i}=L-M-1+i$.

Remark 1. We observe that $f_{\Lambda \mid \mathcal{H}_{0}}\left(\lambda \mid \mathcal{H}_{0}\right)$ only depends on two parameters: the sample size $L$ and the number of antennas $M$, irrespectively of the noise variances. This is a consequence of the power-normalized structure of $\Lambda=|\mathbf{C}|$ as the determinant of the sample coherence matrix $\mathbf{C} \doteq \mathbf{D}^{-1} \mathbf{R} \mathbf{D}^{-1}$, with $\mathbf{D}^{2} \doteq \operatorname{diag}\{\mathbf{R}\}$, which determines that the HRD is a Constant False Alarm Rate (CFAR) detector.

Remark 2. Note that according to the Hadamard's inequality we have $T \geq 1$, and thus $0<$ $\Lambda \leq 1$, which means the support of the PDF of $\Lambda$ is $\lambda \in(0,1]$. This fact is confirmed by (16) as $G_{M-1, M-1}^{M-1,0}\left(\begin{array}{c}a_{1}, \ldots, a_{M-1} \\ b_{1}, \ldots, b_{M-1}\end{array} \mid \lambda\right)=0$ for $|\lambda|>1$ according to Meijer's G-function properties [36].

Therefore, the exact expression of false-alarm probability is obtained as $P_{\mathrm{fa}}=P\left(t>\eta \mid \mathcal{H}_{0}\right)=$ $P\left(\lambda<\frac{1}{\eta} \mid \mathcal{H}_{0}\right)$, with,

$$
P_{\mathrm{fa}}=\frac{(\Gamma(L))^{M}}{\prod_{i=1}^{M} \Gamma(L-M+i)} \int_{0}^{\frac{1}{\eta}} G_{M-1, M-1}^{M-1,0}\left(\begin{array}{c}
a_{1}, \ldots, a_{M-1} \\
b_{1}, \ldots, b_{M-1}
\end{array} \mid \lambda\right) \mathrm{d} \lambda
$$

The above equation can be simplified by using the integration properties of Meijer's G-function [36] 
as,

$$
\begin{aligned}
& P_{\mathrm{fa}}=\frac{(\Gamma(L))^{M}}{\prod_{i=1}^{M} \Gamma(L-M+i)} \\
& \times\left[G_{M, M}^{M-1,1}\left(\begin{array}{c|c}
1, a_{1}+1, \ldots, a_{M-1}+1 & \frac{1}{\eta} \\
b_{1}+1, \ldots, b_{M-1}+1,0 & n
\end{array}\right)-G_{M, M}^{M-1,1}\left(\begin{array}{c}
1, a_{1}+1, \ldots, a_{M-1}+1 \\
b_{1}+1, \ldots, b_{M-1}+1,0
\end{array} \mid 0\right)\right],
\end{aligned}
$$

where the decision threshold, i.e., $\eta$, can be found by numerically solving for $\eta$ in the equation above.

\section{B. Tight approximation to the false-alarm probability}

The exact expression of the false-alarm probability in (17), although theoretically interesting, is difficult to interpret or manipulate. Thus, alternatively, we aim to approximate the distribution of $T$ with a known distribution by fitting its first few moments. For a tight approximation, we use the Log-Gamma distribution, motivated by the following two facts: (a) It is defined on the same support $[1, \infty)$ as $T$; (b) The statistic $\log T$ asymptotically follows a chi-squared distribution [23]; thus, given that the chi-squared distribution is a particular instance of the Gamma distribution, we adopt Gamma as the approximating distribution of $\log T$, so that $T$ will be modeled (asymptotically) by a log-Gamma distribution.

The PDF and Complementary Cumulative Distribution Function (CCDF) of a Log-Gamma distribution with the shape parameter $\alpha_{0}$ and inverse scale parameter $\beta_{0}$ are given by

$$
\begin{aligned}
f_{T}(t) & =\frac{(\ln (t))^{\alpha_{0}-1}}{t \beta_{0}{ }^{\alpha_{0}} \Gamma\left(\alpha_{0}\right)} \exp \left(-\frac{\ln (t)}{\beta_{0}}\right), 1<t<\infty . \\
F_{T}(x) & =P(t>x)=\frac{\Gamma\left(\alpha_{0}, \frac{\ln x}{\beta_{0}}\right)}{\Gamma\left(\alpha_{0}\right)},
\end{aligned}
$$

where the parameters $\alpha_{0}$ and $\beta_{0}$ can be obtained by fitting the two first moments of the LogGamma distribution to the two first moments of $T$. We continue by introducing Lemma .

Lemma 2. The $m^{\text {th }}$ moment of $T$ under $\mathcal{H}_{0}$, where $m \in \mathbb{N}$, is equal to,

$$
\mathrm{E}\left\{T^{m} \mid \mathcal{H}_{0}\right\}=\frac{\Gamma_{\mathrm{M}}(L-m)}{\Gamma_{\mathrm{M}}(L)}\left(\frac{\Gamma(L)}{\Gamma(L-m)}\right)^{M} .
$$

where $\Gamma_{M}(L)=\pi^{\frac{1}{2} M(M-1)} \prod_{j=1}^{M} \Gamma(L-j+1)$.

Proof: Set $z=-m$ and $\Lambda=1 / T$ in equation (8) or set $p=-m$ in [24, Eq. (20)]. 
Using Lemma 2 and $\Gamma(s+1)=s \Gamma(s)$, we obtain the mean, second moment and variance of $T$ under $\mathcal{H}_{0}$,

$$
\begin{aligned}
\mu_{T \mid \mathcal{H}_{0}} & \doteq \mathrm{E}\left\{T \mid \mathcal{H}_{0}\right\}=\prod_{j=1}^{M} \frac{L-1}{L-j} \\
\mu_{2, T \mid \mathcal{H}_{0}} & \doteq \mathrm{E}\left\{T^{2} \mid \mathcal{H}_{0}\right\}=\frac{\Gamma_{\mathrm{M}}(L-2)}{\Gamma_{\mathrm{M}}(L)}\left(\frac{\Gamma(L)}{\Gamma(L-2)}\right)^{M} \\
\sigma_{T \mid \mathcal{H}_{0}}^{2} & \doteq \mu_{2, T \mid \mathcal{H}_{1}}-\mu_{T \mid \mathcal{H}_{0}}^{2} \\
& =\mu_{T \mid \mathcal{H}_{0}}^{2} \cdot\left(\frac{L-1}{L-M-1}\left(\frac{L-2}{L-1}\right)^{M}-1\right)
\end{aligned}
$$

which, as in (16), are functions of only $L$ and $M$. These expressions let us verify that:

1) $\mu_{T \mid \mathcal{H}_{0}} \geq 1$ for any $L, M$, and, asymptotically: $\lim _{L \rightarrow \infty} \mu_{T \mid \mathcal{H}_{0}}=1$ (for finite $M$ ), as the sample correlation matrix $\mathbf{R}$ tends to the true diagonal correlation $\boldsymbol{\Sigma}$ under $\mathcal{H}_{0}$ so that $T \rightarrow 1^{+}$.

2) The ratio $\mu_{T \mid \mathcal{H}_{0}}^{2} / \sigma_{T \mid \mathcal{H}_{0}}^{2}$ goes to infinity at $L \rightarrow \infty$, as expected when the sample correlations in $T$ converge.

Now, by setting $\mu_{T \mid \mathcal{H}_{0}}$ and $\sigma_{T \mid \mathcal{H}_{0}}^{2}$ equal to the mean and variance of the Log-Gamma distribution, respectively, we have from $\alpha_{0}$ and $\beta_{0}$ in $(19 \mathrm{a}, 19 \mathrm{~b})$,

$$
\begin{array}{ll}
\mu_{T \mid \mathcal{H}_{0}}=\left(1-\beta_{0}\right)^{-\alpha_{0}} & , 0<\beta_{0}<1, \\
\sigma_{T \mid \mathcal{H}_{0}}^{2} & =\left(1-2 \beta_{0}\right)^{-\alpha_{0}}-\left(1-\beta_{0}\right)^{-2 \alpha_{0}}, 0<\beta_{0}<1 / 2
\end{array}
$$

To solve for $\beta_{0}$ and $\alpha_{0}$, let us define $g(\beta)$ and its inverse function $g^{-1}(\cdot)$ (which can be evaluated numerically),

$$
g(\beta)=\frac{\ln (1-\beta)}{\ln (1-2 \beta)} \quad, \quad 0 \leq \beta \leq \frac{1}{2}
$$

$g(\beta)$ is convex- $\cap$ and decreasing, mapping $\beta \in\left\{0, \frac{1}{2}\right\}$ to $g \in\left\{\frac{1}{2}, 0\right\}$. Hence,

$$
\begin{aligned}
& \alpha_{0}=\frac{\ln \left(\mu_{T \mid \mathcal{H}_{0}}\right)}{-\ln \left(1-\beta_{0}\right)} \\
& \beta_{0}=g^{-1}(p) \quad, \quad p \doteq \frac{1}{2} \cdot \frac{\ln \left(\mu_{T \mid \mathcal{H}_{0}}^{2}\right)}{\ln \left(\mu_{T \mid \mathcal{H}_{0}}^{2}+\sigma_{T \mid \mathcal{H}_{0}}^{2}\right)}
\end{aligned}
$$

Therefore, as $\mu_{T \mid \mathcal{H}_{0}} \geq 1$, we note that $p$ is related to the concentration of the test $T$ about its expected value $\mu_{T \mid \mathcal{H}_{0}}$, with $0 \leq p \leq \frac{1}{2}$ and $p$ increasing for higher concentration so that, 
asymptotically $(L \rightarrow \infty)$, we have $p \rightarrow \frac{1}{2}^{-}$. Accordingly, the parameter $\beta_{0}$ determines the amount of dispersion of the test $T$ about its expected value $\mu_{T \mid \mathcal{H}_{0}}$ such that increase in $\beta_{0}$ results in more dispersion of the test $T$ about its expected value and vice versa. In addition, with respect to (27), the parameter $\alpha_{0}$ is relevant to the ratio of the mean to dispersion of $T$ and, at a fixed $\beta_{0}$, increase in $\alpha_{0}$ is associated with increase in the mean of the test $T$.

Finally, the false-alarm probability for the HRD based on this approximate distribution of $T$ and its equivalent decision threshold $\eta$ are obtained as,

$$
\begin{aligned}
P_{\mathrm{fa}} & =P\left(t>\eta \mid \mathcal{H}_{0}\right) \approx F_{T}(\eta) . \\
\eta & \approx F_{T}^{-1}\left(P_{\mathrm{fa}}\right) .
\end{aligned}
$$

with $F_{T}^{-1}($.$) the inverse function of F_{T}(x)$, which can also be obtained numerically. Let us define an associated measure of dispersion,

$$
\sigma_{p}^{2} \doteq 1-2 p=\frac{\ln \left(1+\sigma_{T \mid \mathcal{H}_{0}}^{2} / \mu_{T \mid \mathcal{H}_{0}}^{2}\right)}{\ln \left(\mu_{T \mid \mathcal{H}_{0}}^{2}+\sigma_{T \mid \mathcal{H}_{0}}^{2}\right)}
$$

with $\sigma_{p}^{2} \rightarrow 0^{+}$as $L \rightarrow \infty$. Now, more specific expressions for $\alpha_{0}$ and $\beta_{0}$ are possible if we turn to a large- $L$ approximation where $\sigma_{p}^{2}<<1$. These large- $L$ approximations let us intuitively assess the impact of $M$ and $L$ on the false-alarm probability. Let us consider the approximation $g(x) \simeq \frac{1}{2}-\frac{1}{4} x-\frac{1}{4} x^{2}$ at $x \rightarrow 0^{+}\left(g(x) \rightarrow \frac{1}{2}^{-}\right)$. Thus, for $\sigma_{p}^{2}<<1$ where $\beta_{0}<<1$, we may use $\beta_{0}=g^{-1}(p)=2 \sigma_{p}^{2}-4 \sigma_{p}^{4}+o\left(\sigma_{p}^{4}\right)$, and $\frac{1}{\beta_{0}}$ can be approximated as,

$$
\frac{1}{\beta_{0}}=\frac{1}{2 \sigma_{p}^{2}}+1+o\left(\sigma_{p}^{2}\right)
$$

As for small $\beta_{0}$ we have $\frac{-1}{\ln \left(1-\beta_{0}\right)}=\frac{1}{\beta_{0}}-\frac{1}{2}+o\left(\beta_{0}\right)$, the following approximation results for $\alpha_{0}$,

$$
\alpha_{0} \simeq\left(\frac{1}{\beta_{0}}-\frac{1}{2}\right) \ln \mu_{T \mid \mathcal{H}_{0}}
$$

The final large- $L$ approximations for $\alpha_{0}$ and $\beta_{0}$ are summarized in table I from the results in appendix D. Fig. 1 investigates the accuracy of large- $L$ approximations compared to the true values and shows a good agreement between them even for moderate values of $L$. Considering that $F_{T}(\eta)$ is an increasing function with respect to parameters $\alpha_{0}$ and $\beta_{0}$, and also considering the large- $L$ approximations of $\alpha_{0}$ and $\beta_{0}$ given in table I, we can intuitively investigate the impact of $M$ and $L$ on the false-alarm probability. Accordingly, for a fixed decision threshold, the false-alarm probability decreases by increasing $L$ or decreasing $M$. Equivalently, considering 
TABLE I

LARGE- $L$ APPROXIMATIONS TO THE SHAPE PARAMETER AND INVERSE SCALE PARAMETER OF THE APPROXIMATING LOG-GAMMA PDF UNDER $\mathcal{H}_{0}$ AND $\mathcal{H}_{1}$

\begin{tabular}{|c|c|}
\hline Weighting term & Large- $L$ approximation \\
\hline$\alpha_{0}$ & $\frac{1}{2} M(M-1)$ \\
\hline$\beta_{0}^{-1}$ & $L-\frac{M+1}{3}$ \\
\hline$\alpha_{1}$ & $\left(\beta_{1}^{-1}-\frac{1}{2}\right)\left(\frac{M(M-1)+\Delta}{2 L}+\ln \frac{1}{\left|\mathbf{C}_{Q}\right|}\right)$ \\
& $\rightarrow L \cdot \frac{1}{4 \Delta} \ln ^{2} \frac{1}{\left|\mathbf{C}_{Q}\right|^{2}},(L \rightarrow \infty)$ \\
\hline$\beta_{1}^{-1}$ & $\begin{array}{c}\frac{1}{2 \Delta}\left(M(M-1)+2 \Delta+L \ln \frac{1}{\left|\mathbf{C}_{Q}\right|^{2}}\right)+1 \\
\rightarrow L \cdot \frac{1}{2 \Delta} \ln \frac{1}{\left|\mathbf{C}_{Q}\right|^{2}},(L \rightarrow \infty)\end{array}$ \\
\hline Definitions: & $\begin{array}{c}\mathbf{D}_{Q}^{2} \doteq \operatorname{diag}\{\mathbf{Q}\}, \mathbf{C}_{Q} \doteq \mathbf{D}_{Q}^{-1} \mathbf{Q} \mathbf{D}_{Q}^{-1}, \\
\end{array}$ \\
\hline
\end{tabular}
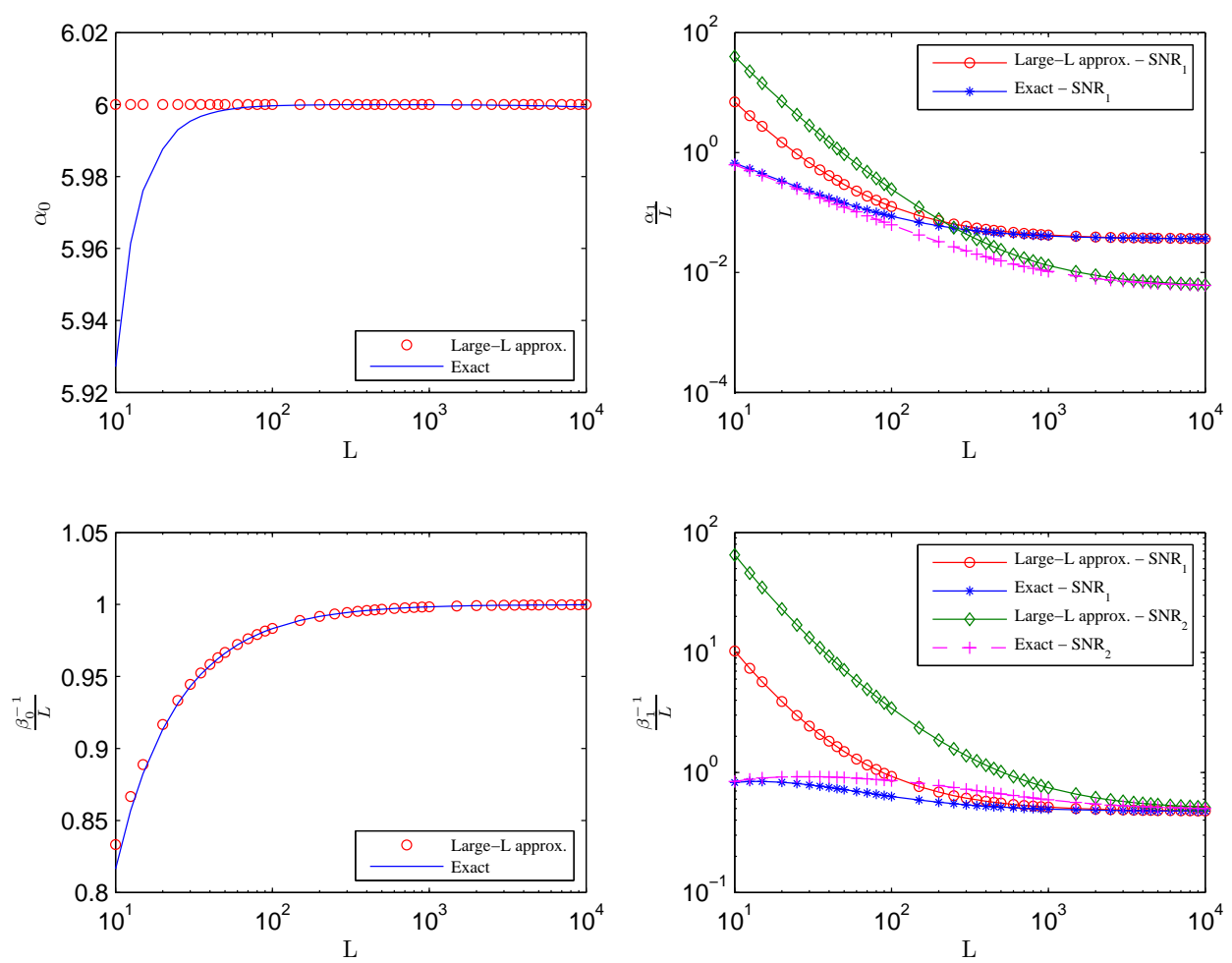

Fig. 1. Large- $L$ approximations for $M=4$ antennas. Top left: $\alpha_{0}$; Bottom left: $\frac{1}{L} \beta_{0}^{-1}$; Top-right: $\frac{1}{L} \alpha_{1}$ at $\mathrm{SNR}_{1}=$ $0 d B, \mathrm{SNR}_{2}=-6 d B$; Bottom-right: $\frac{1}{L} \beta_{1}^{-1}$ at $\mathrm{SNR}_{1}=0 d B, \mathrm{SNR}_{2}=-6 d B$.

that $F_{T}(\eta)$ is a decreasing function with respect to $\eta$, we could say that for a fixed false-alarm probability, the decision threshold decreases by increasing $L$ or decreasing $M$. http://www.ieee.org/publications_standards/publications/rights/index.html for more information. 


\section{Detection Probability}

In general, we can not derive the exact distribution of $T$ under $\mathcal{H}_{1}$ by using the inverse Mellin transform because, unlike $\mathcal{H}_{0}$, the random variable $\prod_{i=1}^{M} r_{i, i}$ is not independent of the decision statistic $T$ and thus it does not seem possible to obtain the complex moments of $T$ under $\mathcal{H}_{1}$. But, as for $\mathcal{H}_{0}$, we can derive a tight approximation to the distribution of $T$ under $\mathcal{H}_{1}$ by approximating the distribution of $T$ with a known distribution that fits its first few moments. As in the previous section, we approximate the distribution of $T$ under $\mathcal{H}_{1}$ with a Log-Gamma distribution with the shape parameter $\alpha_{1}$ and inverse scale parameter $\beta_{1}$ by fitting its two first moments.

Theorem 1. The $m^{\text {th }}$ moment of $T$ under $\mathcal{H}_{1}$, where $m \in \mathbb{N}$, is equal to,

$$
\mathrm{E}\left\{T^{m} \mid \mathcal{H}_{1}\right\}=\frac{\Gamma_{\mathrm{M}}(L-m)}{\Gamma_{\mathrm{M}}(L)|\mathbf{Q}|^{m}} \sum_{k=1}^{m M}(L-m)^{k} \psi_{m M, k},
$$

with $\psi_{m M, k}$ is defined as,

$$
\psi_{m M, k}=\sum_{\pi \in \mathcal{I}_{m M}} \prod_{p=1}^{m M} q_{i_{p}, i_{\pi(p)}}
$$

where $\mathcal{I}_{m M}$ is the subgroup of symmetric group ${ }^{1} \mathcal{S}_{m M}$ on the finite set $\{1,2,3, \cdots, m M\}$ including all the permutations $\pi$ which are permutated to the identity permutation exactly with $(m M-k)$ permutations and $\left(i_{1}, i_{2}, \cdots, i_{m M}\right)=\left(\left(\mathbf{1}_{m}\right),\left(\mathbf{2}_{m}\right), \cdots,\left(\mathbf{M}_{m}\right)\right)^{2}$.

Proof: See Appendix B.

Remark 3. Note that the coefficients $\psi_{m M, k}$ do not depend on the number of samples per antenna $L$ and that the complexity of computing $\psi_{m M, k}$ increases as $k$ decreases from $m M$ to 1 . Thus for the case that the number of samples per antenna $L$ is large enough we could use a truncated form of (34) with the most relevant coefficients $\psi_{m M, k}$, which has lower computational complexity compared to (34), as,

$$
\mathrm{E}\left\{T^{m} \mid \mathcal{H}_{1}\right\} \simeq \frac{\Gamma_{\mathrm{M}}(L-m)}{\Gamma_{\mathrm{M}}(L)|\mathbf{Q}|^{m}} \sum_{k=b}^{m M}(L-m)^{k} \psi_{m M, k},
$$

\footnotetext{
${ }^{1}$ The symmetric group $\mathcal{S}_{n}$ on a finite set of $n$ symbols is the group whose elements are all the permutations of the $n$ symbols, and whose group operation is the composition of such permutations.

${ }^{2}$ The notation $\left(\varphi_{n}\right)$ is used to denote a $n$-tuple whose elements are all $\varphi$ 's.
} 
with $b>1$ such that $\left|\frac{\Gamma_{\mathrm{M}}(L-m)}{\Gamma_{\mathrm{M}}(L)|\mathbf{Q}|^{m}}\left(\sum_{k=1}^{m M}(L-m)^{k} \psi_{m M, k}-\sum_{k=b}^{m M}(L-m)^{k} \psi_{m M, k}\right)\right|$ is negligible.

From theorem 1, the mean, second moment and variance of $T$ under $\mathcal{H}_{1}$ are derived as,

$$
\begin{aligned}
\mu_{T \mid \mathcal{H}_{1}} & \doteq \mathrm{E}\left\{T \mid \mathcal{H}_{1}\right\}=\frac{\Gamma_{\mathrm{M}}(L-1)}{\Gamma_{\mathrm{M}}(L)|\mathbf{Q}|} \sum_{k=1}^{M}(L-1)^{k} \psi_{M, k} \\
\mu_{2, T \mid \mathcal{H}_{1}} & \doteq \mathrm{E}\left\{T^{2} \mid \mathcal{H}_{1}\right\}=\frac{\Gamma_{\mathrm{M}}(L-2)}{\Gamma_{\mathrm{M}}(L)|\mathbf{Q}|^{2}} \sum_{k=1}^{2 M}(L-2)^{k} \psi_{2 M, k} \\
\sigma_{T \mid \mathcal{H}_{1}}^{2} & \doteq \mu_{2, T \mid \mathcal{H}_{1}}-\mu_{T \mid \mathcal{H}_{1}}^{2}
\end{aligned}
$$

Computing the most relevant coefficients $\psi_{m M, k}$ in (37) and (39) according to remark 3 contributes later to gaining insight as to how signal parameters affect the performance of the HRD. The remaining coefficients only constitute a refinement and are hardly useful for interpretation. Applying (35) and defining the diagonal matrix $\mathbf{D}_{Q}^{2} \doteq \operatorname{diag}\{\mathbf{Q}\}$ featuring the power at each antenna as well as the coherence matrix $\mathbf{C}_{Q} \doteq \mathbf{D}_{Q}^{-1} \mathbf{Q} \mathbf{D}_{Q}^{-1}$, the coefficients $\psi_{M, M}$ and $\psi_{M, M-1}(m=1)$ and $\psi_{2 M, 2 M}$ and $\psi_{2 M, 2 M-1}(m=2)$ are computed in appendix C. Therefore, setting $b=m M-1$ in (36), we get the following second-order approximation for large $L$ to the first two moments of $T$ (see appendix C),

$$
\begin{aligned}
\mu_{T \mid \mathcal{H}_{1}} & =\left(\prod_{j=1}^{M} \frac{L-1}{L-j}\right) \cdot \frac{1}{\left|\mathbf{C}_{Q}\right|}\left(1+\frac{\left\|\mathbf{C}_{Q}-\mathbf{I}_{M}\right\|_{F}^{2}}{2(L-1)}+o\left(\frac{1}{L}\right)\right) \\
\mu_{2, T \mid \mathcal{H}_{1}} & =\left(\prod_{j=1}^{M} \frac{L-2}{L-j}\right)^{2} \frac{L-1}{L-M-1} \cdot \frac{1}{\left|\mathbf{C}_{Q}\right|^{2}}\left(1+\frac{M+2\left\|\mathbf{C}_{Q}-\mathbf{I}_{M}\right\|_{F}^{2}}{L-2}+o\left(\frac{1}{L}\right)\right)
\end{aligned}
$$

In addition, the second-order approximation to $\sigma_{T \mid \mathcal{H}_{1}}^{2}$ is obtained straightforwardly by substituting (40) and (41) in (39). So, the two first moments used for fitting the distribution of $T$ are controlled by $\left|\mathbf{C}_{Q}\right|$ : the determinant of the coherence matrix under $\mathcal{H}_{1}$ (equal to 1 under $\mathcal{H}_{0}$ ), and in a lesser degree by $\Delta \doteq\left\|\mathbf{C}_{Q}-\mathbf{I}_{M}\right\|_{F}^{2}$ : the energy of its off-diagonal terms (equal to 0 under $\mathcal{H}_{0}$ ). In fact, it is shown in [23] that at low-SNR: $\ln \left|\mathbf{C}_{Q}\right| \simeq-\frac{1}{2}\left(\left\|\mathbf{C}_{Q}\right\|_{F}^{2}-M\right)=-\frac{1}{2}\left\|\mathbf{C}_{Q}-\mathbf{I}_{M}\right\|_{F}^{2}$, which makes the approximation to both moments in (40) and (41) dependent on a single parameter: $\Delta$.

Now, setting $\mu_{T \mid \mathcal{H}_{1}}$ and $\sigma_{T \mid \mathcal{H}_{1}}^{2}$ equal to the mean and variance of the Log-Gamma distribution, 
we can obtain $\beta_{1}$ and $\alpha_{1}$ as follows,

$$
\begin{aligned}
& \alpha_{1}=\frac{\ln \left(\mu_{T \mid \mathcal{H}_{1}}\right)}{-\ln \left(1-\beta_{1}\right)}, \\
& \beta_{1}=g^{-1}(p) \quad, \quad p \doteq \frac{1}{2} \cdot \frac{\ln \left(\mu_{T \mid \mathcal{H}_{1}}^{2}\right)}{\ln \left(\mu_{T \mid \mathcal{H}_{1}}^{2}+\sigma_{T \mid \mathcal{H}_{1}}^{2}\right)}
\end{aligned}
$$

with $g(\beta)$ as in (26). The HRD detection probability based on this approximate distribution can now be obtained as,

$$
P_{\mathrm{d}}=P\left(t>\eta \mid \mathcal{H}_{1}\right) \approx \frac{\Gamma\left(\alpha_{1}, \frac{\ln \eta}{\beta_{1}}\right)}{\Gamma\left(\alpha_{1}\right)} .
$$

It is worth pointing out that the equations (42a) and (42b) have the same form as $(27,28)$, by exchanging $\mathcal{H}_{0}$ with $\mathcal{H}_{1}$. Hence, the same institutive interpretations provided for $\alpha_{0}$ and $\beta_{0}$ are also valid for $\alpha_{1}$ and $\beta_{1}$. Furthermore, the same expressions $(31,32,33)$ can be exploited to obtain the equivalent large- $L$ approximations for parameters $\alpha_{1}$ and $\frac{1}{\beta_{1}}$. The corresponding large- $L$ approximations are provided in table I based on the results in appendix D. Note that the approximations in table I are applicable where $\mathbf{C}_{Q} \neq \mathbf{I}_{M}$ and $L$ is sufficiently large. Otherwise, infinitesimals of order $1 / L^{2}$ and higher in $(40,41)$ will start weighing in. Fig. 1 shows that $\alpha_{1}$ and $\frac{1}{\beta_{1}}$ can be accurately predicted for large values of $L$ by using the mentioned large- $L$ approximations. Note that in this case, in contrast to $\mathcal{H}_{0}$, both parameters $\alpha_{1}$ and $\frac{1}{\beta_{1}}$ increase linearly with $L$. However, as discussed in III-B, increase in $L$ accordingly will decrease the decision threshold which partly counteracts to the impact of increase in $\frac{1}{\beta_{1}}$. Consequently in overall, increase in $L$ will increase the detection probability through increasing $\alpha_{1}$. Using the low-SNR approximate: $\ln \left|\mathbf{C}_{Q}\right| \simeq-\frac{1}{2} \Delta$, the large- $L$ approximations of $\alpha_{1}$ and $\frac{1}{\beta_{1}}$ in table I will tend to the values of $\frac{L \Delta}{4}$ and $\frac{L}{2}$, respectively. Therefore, increase in energy of off-diagonal terms of $\mathrm{Q}$, which is related to the SNR, results to increase in the detection probability through increasing $\alpha_{1}$. Finally as it can be seen although $M$ does not appear in large- $L$ approximations

of $\alpha_{1}$ and $\frac{1}{\beta_{1}}$ explicitly, however indirectly boost the detection probability through: 1) decreasing the decision threshold; and 2) increasing the SNR.

\section{Simulation Results}

We provide Monte Carlo simulations ${ }^{3}$ to verify the proposed exact and approximate closedform expressions for the false-alarm probability $P_{\mathrm{fa}}$ and the proposed closed-form approximation

\footnotetext{
${ }^{3}$ For each simulated point $10^{6}$ Monte Carlo repetitions has been computed.
} 


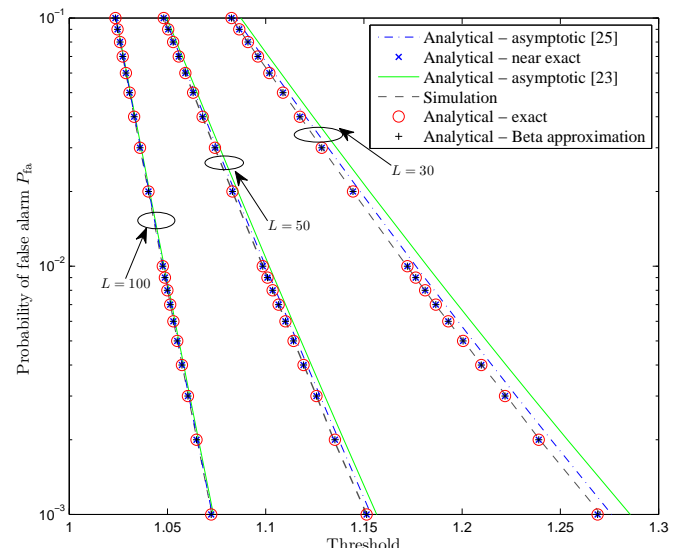

(a) $M=2$

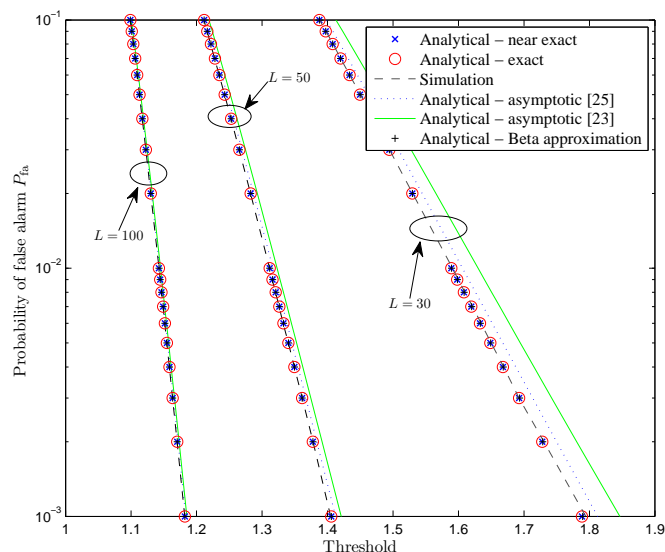

(b) $M=4$

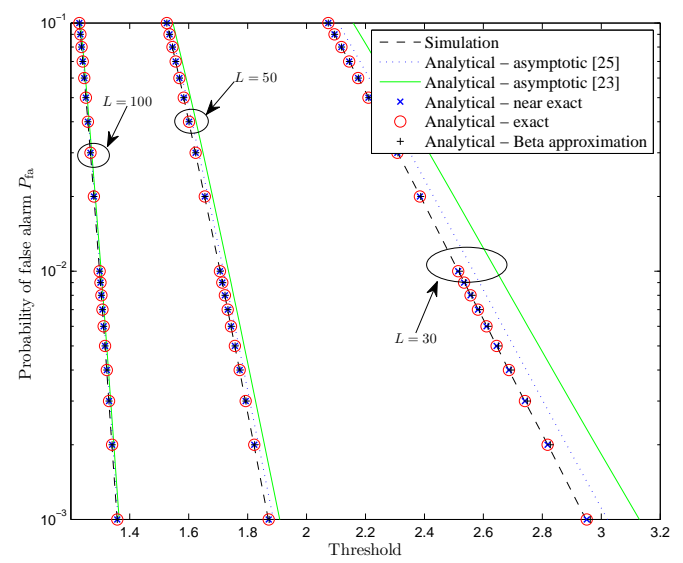

(c) $M=6$

Fig. 2. False-alarm probability versus decision threshold for different values of $L$ and $M$.

to the detection probability $P_{\mathrm{d}}$. We also compare the closed-form expressions for $P_{\mathrm{fa}}$ and $P_{\mathrm{d}}$ with previously reported approximations.

\section{A. False-Alarm Probability versus Threshold}

Fig. 2 plots the false-alarm probability versus the threshold for different values of $M$ and $L$ in which we compare the derived exact and approximate closed-form expressions for the false-alarm probability with each other, with the asymptotic closed-form expressions in [23], [25] and with the moment-based beta approximation in [24]. The per-antenna noise variances are set at: $(0,-1,1,0.5,-1,0) \mathrm{dB}$ for $M=6,(0,-1,1,0.5) \mathrm{dB}$ for $M=4$, and $(0,-1) \mathrm{dB}$ for $M=2$. A great closeness can be observed between the exact and approximate closed-form expressions for the probability of false alarm, although the latter is much less complicated. In 
TABLE II

THE ANAD VALUES FOR THE DIFFERENT ANALYTICAL EXPRESSIONS

\begin{tabular}{|c|c|c|c|c|c|c|c|c|c|}
\hline \multirow{3}{*}{ Related analytical expression } & \multicolumn{9}{|c|}{ values of $M$ and $L$} \\
\hline & $M=2$ & $M=2$ & $M=2$ & $M=4$ & $M=4$ & $M=4$ & $M=6$ & $M=6$ & $M=6$ \\
\hline & $L=30$ & $L=50$ & $L=100$ & $L=30$ & $L=50$ & $L=100$ & $L=30$ & $L=50$ & $L=100$ \\
\hline exact & $6.37 \times 10^{-6}$ & $5.09 \times 10^{-6}$ & $4.73 \times 10^{-6}$ & $5.53 \times 10^{-6}$ & $6.86 \times 10^{-6}$ & $6.31 \times 10^{-6}$ & $6.35 \times 10^{-6}$ & $5.67 \times 10^{-6}$ & $6.24 \times 10^{-6}$ \\
\hline approx. & $2.03 \times 10^{-4}$ & $1.91 \times 10^{-4}$ & $1.89 \times 10^{-4}$ & $1.94 \times 10^{-4}$ & $1.98 \times 10^{-4}$ & $1.83 \times 10^{-4}$ & $1.75 \times 10^{-4}$ & $1.82 \times 10^{-4}$ & $1.93 \times 10^{-4}$ \\
\hline beta approximation [24] & $4.90 \times 10^{-4}$ & $1.63 \times 10^{-4}$ & $3.12 \times 10^{-4}$ & $2.09 \times 10^{-4}$ & $2.27 \times 10^{-4}$ & $1.54 \times 10^{-4}$ & $1.95 \times 10^{-4}$ & $3.15 \times 10^{-4}$ & $2.48 \times 10^{-4}$ \\
\hline asymptotic [25] & $4.72 \times 10^{-3}$ & $1.06 \times 10^{-3}$ & $3.52 \times 10^{-4}$ & $1.24 \times 10^{-2}$ & $4.13 \times 10^{-3}$ & $9.84 \times 10^{-4}$ & $4.96 \times 10^{-2}$ & $7.47 \times 10^{-3}$ & $2.16 \times 10^{-3}$ \\
\hline asymptotic [23] & $1.11 \times 10^{-2}$ & $3.6 \times 10^{-3}$ & $7.81 \times 10^{-4}$ & $2.95 \times 10^{-2}$ & $9.53 \times 10^{-3}$ & $2.4 \times 10^{-3}$ & $1.09 \times 10^{-1}$ & $1.68 \times 10^{-2}$ & $4.6 \times 10^{-3}$ \\
\hline
\end{tabular}

addition, it can be seen that the approximate closed-form expression and the beta approximation in [24] have roughly the same accuracy, but, the former is more accurate in comparison with the previously reported asymptotic closed-form expressions in [23] and [25]. We also show, quantitatively, the accuracy improvement of the exact and approximate closed-form expressions over the previously reported asymptotic closed-form expressions in [23] and [25] in terms of the Average Normalized Absolute Difference (ANAD) between the threshold computed by each of the analytical expressions and the true value of the threshold obtained from Monte Carlo simulation ${ }^{4}$. The results are shown in Table $\mathrm{II}^{5}$. It can be seen that the accuracy of the exact and approximate closed-form expressions is not affected by $L$ and $M$, while the accuracy of the asymptotic closed-form expressions in [23] and [25] improves with increasing $L$ and decreasing $M$.

\section{B. Detection Probability versus SNR}

Fig. 3 shows the probability of missed detection, i.e. $P_{\mathrm{m}}=1-P_{\mathrm{d}}$, versus SNR at $P_{\mathrm{fa}}=0.01$, $M=4, K=2$ and $L=1000$, for three cases in which the PU system uses, respectively: (a) a 16-PSK modulation; (b) a 16-QAM modulation; (c) a 64-QAM modulation. We consider square-root raised-cosine pulses at 0.25 roll-off truncated to 24 symbols in length. Moreover, for each (a), (b) or (c), we consider two sampling rates for the received signal: $N_{s s}=1$ and $N_{s s}=2$ samples per symbol. The per-antenna noise variances equal $(0,-1,1,0.5) \mathrm{dB}$. To test the expressions for as large a set of instances of $\mathbf{H}$ as possible, for each Monte Carlo simulation,

${ }^{4}$ The ANAD is defined as $\frac{1}{p} \sum_{i=1}^{p} \frac{\left|\eta_{A}\left(P_{\mathrm{fa}_{\mathrm{i}}}\right)-\eta_{S}\left(P_{\mathrm{fa}_{\mathrm{i}}}\right)\right|}{\eta_{S}\left(P_{\mathrm{fa}_{\mathrm{i}}}\right)}$ where $\eta_{A}\left(P_{\mathrm{fa}_{\mathrm{i}}}\right)$ and $\eta_{S}\left(P_{\mathrm{fa}_{\mathrm{i}}}\right)$ are the thresholds evaluated for the false-alarm rate of $P_{\mathrm{fa}_{\mathrm{i}}}$ by one of the analytical expressions and Monte Carlo simulation, respectively.

${ }^{5}$ The ANAD values in Table II have been evaluated for these false-alarm rates $\left\{(1,2, \cdots, 9) \times 10^{-4},(1,2, \cdots, 9) \times\right.$ $\left.10^{-3},(1,2, \cdots, 9) \times 10^{-2},(1,2, \cdots, 9) \times 10^{-1}\right\}$. 


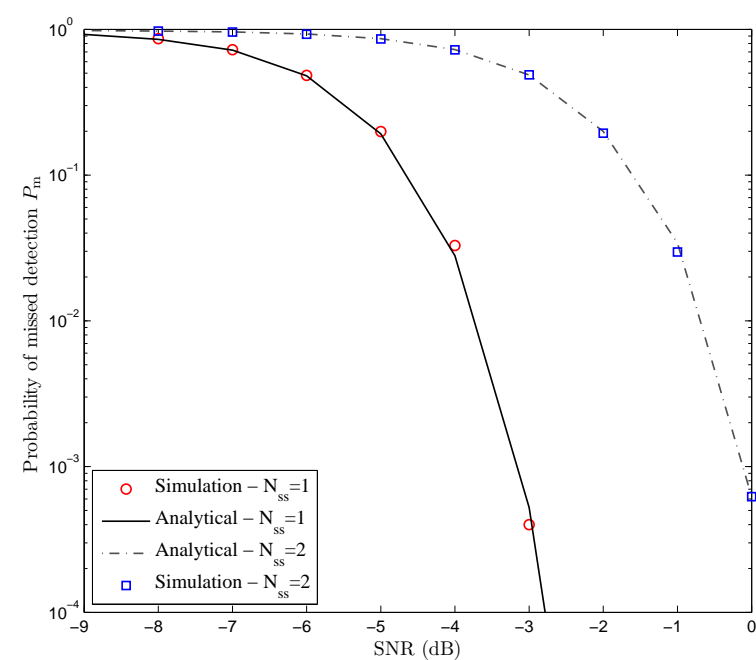

(a)

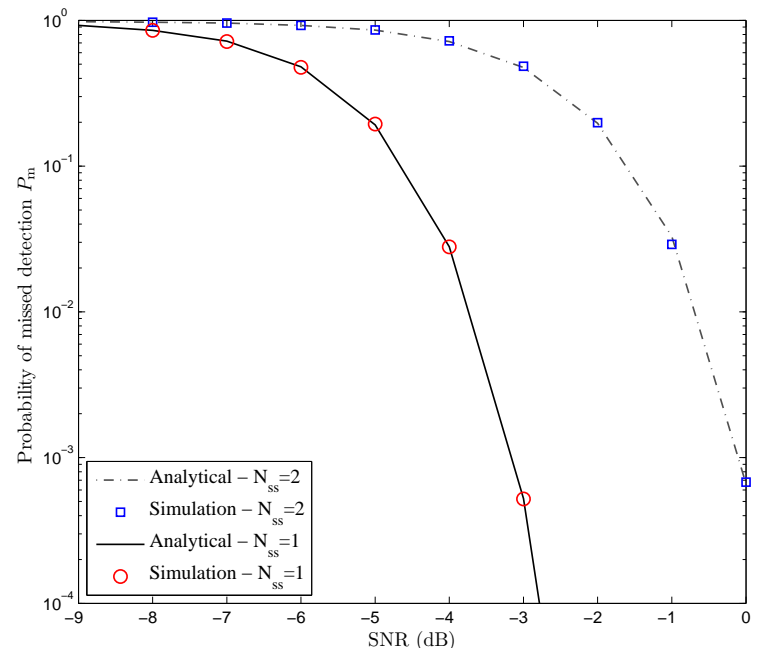

(b)

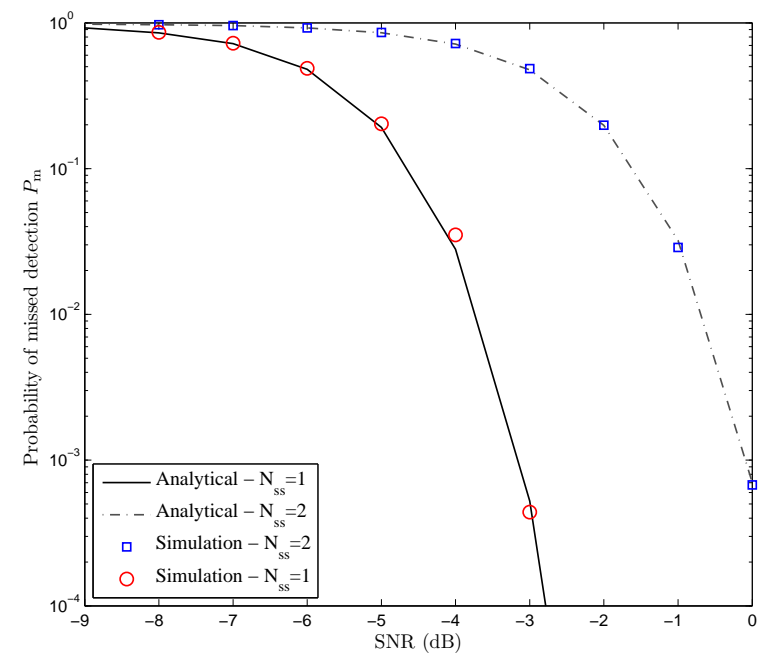

(c)

Fig. 3. Missed detection probability versus SNR for the HRD at $P_{\mathrm{fa}}=10^{-2}, L=1000, M=4$ and $K=2$ : (a) 16-PSK modulation scheme (b) 16-QAM modulation scheme (c) 64-QAM modulation scheme.

we have generated the channel gains matrix $\mathbf{H}$ once by drawing its elements independently from a Gaussian distribution. Then, this matrix is used unchanged in all system realizations within that Monte Carlo simulation. Additionally, the channel gains are scaled to achieve the desired SNR over all Monte Carlo simulations. The SNR is defined as,

$$
\mathrm{SNR} \doteq \operatorname{tr}\left(\mathbf{H} \boldsymbol{\Sigma}^{-1} \mathbf{H}^{H}\right)
$$

The analytical performance is obtained by using the approximate closed-form expressions for computing both the threshold and the detection probability. A great closeness is observed between 
those approximations and Monte Carlo simulations for the different modulation types in all values of SNR even when the received signal is sampled at $N_{s s}=2$. Note that the performance degradation at $N_{s s}=2$ with respect to $N_{s s}=1$ results from the decrease in the SNR due to oversampling $^{6}$. Fig. 3 concludes that the proposed closed-form expressions for false-alarm and detection probabilities are still valid and accurate when the actual distribution of the PU signal is not Gaussian. This may be related to the fact that the HRD operates on the sample covariance matrix R, whose entries, when the data have finite fourth-order moments [29]-[31] and for a sufficiently large sample size $L$, converge to a Gaussian distribution even though the distribution of the PU be not, necessarily, Gaussian. So, the Gaussian assumption for the PU is not a limiting factor in our derivation. Results for different modulations have been shown in different sub-figures due to the closeness of the corresponding curves. It is known that oversampling creates temporal correlation between the PU signal samples. Further, Fig. 3 shows that at lowrate oversampling for which the detection window spans many temporal correlation lengths (i.e. $L>>N_{s s}$ ), the proposed analytical expressions for the performance of the HRD are still valid and accurate. Obviously, if the oversampling rate were increased further (keeping $L$ fixed), the good agreement between the true performance of the HRD and the proposed expressions might be penalized.

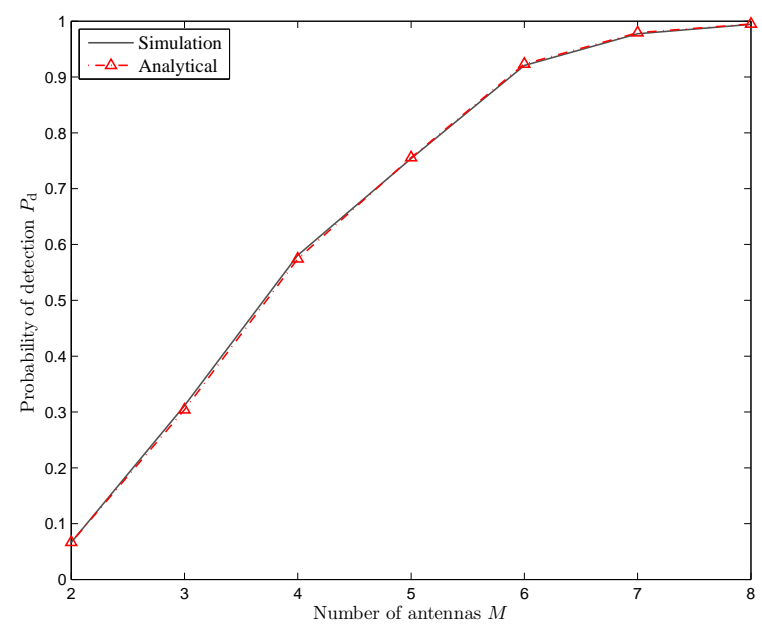

Fig. 4. Detection probability versus the number of antennas $M$ for the HRD at $P_{\mathrm{fa}}=0.01, K=2$ and $L=1000$.

\footnotetext{
${ }^{6}$ Oversampling by 2 doubles the sampling bandwidth and thus the noise power without practically changing the signal power. Accordingly, the SNR decreases by $3 \mathrm{~dB}$ in case $N_{s s}=2$ in comparison to case $N_{s s}=1$.
} 


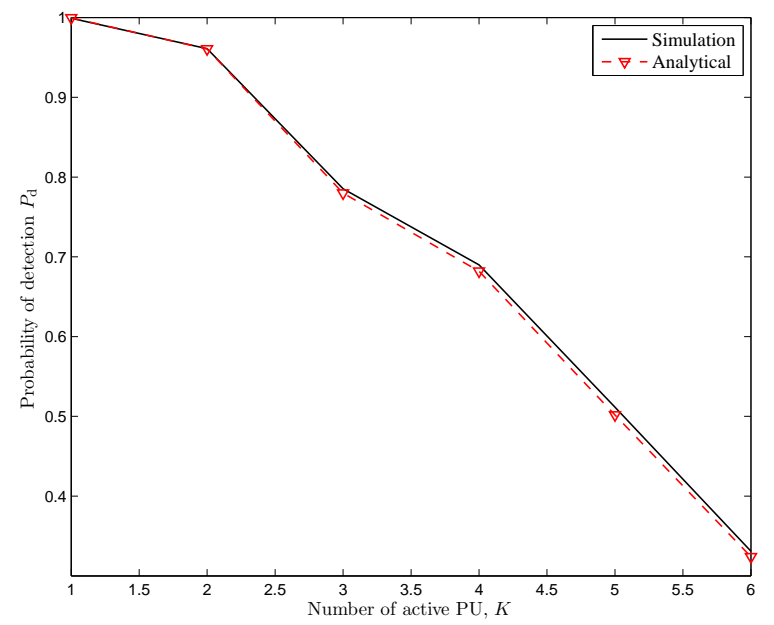

Fig. 5. Detection probability versus the number of active PUs $K$ for the HRD at $S N R=-5 \mathrm{~dB}, M=6$ and $L=1000$.

\section{Detection Probability versus Number of receiving antennas}

Fig. 4 plots the probability of detection versus the number of antennas, $M$, at $P_{\mathrm{fa}}=0.01 \mathrm{~dB}$, $S N R=-12 \mathrm{~dB}, K=2$ and $L=1000$ when the PU system uses a 64-QAM modulation with square-root raised-cosine pulses at 0.25 roll-off truncated to 24 symbols in length and the received signal sampled at $N_{s s}=1$. The elements of $\mathbf{H}$ are generated as in section V-B. The per-antenna noise variances equal $(0,-1,1,0.5,-1,0,0.75,0.25) \mathrm{dB}$ for $M=8,(0,-1,1,0.5,-1,0) \mathrm{dB}$ for $M=6,(0,-1,1,0.5) \mathrm{dB}$ for $M=4$, and $(0,-1) \mathrm{dB}$ for $M=2$. As expected, the performance of the HRD improves when $M$ increases. We also note a very good agreement over $M$ between the approximate closed-form expression and Monte Carlo simulation.

\section{Detection Probability versus Number of Active PUs}

Fig. 5 plots the probability of detection versus the number of active PUs, $K$, at $P_{\mathrm{fa}}=0.01$ $\mathrm{dB}, S N R=-12 \mathrm{~dB}, M=4$ and $L=1000$ when the PU system uses a DQPSK modulation with square-root raised-cosine pulses at 0.25 roll-off truncated to 24 symbols in length. The perantenna noise variances equal $(0,-1,1,0.5) \mathrm{dB}$. The other parameters are those in the previous section. As can be seen, the performance of the HRD degrades when the number of active PUs grows. Furthermore, we observe that simulation results are very close to the analytical-based performance for different values of $K$. 


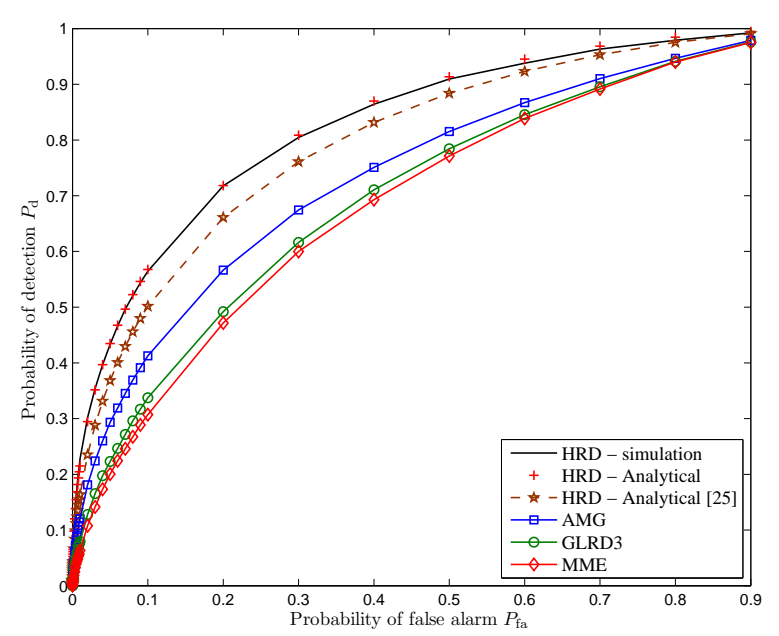

(a)

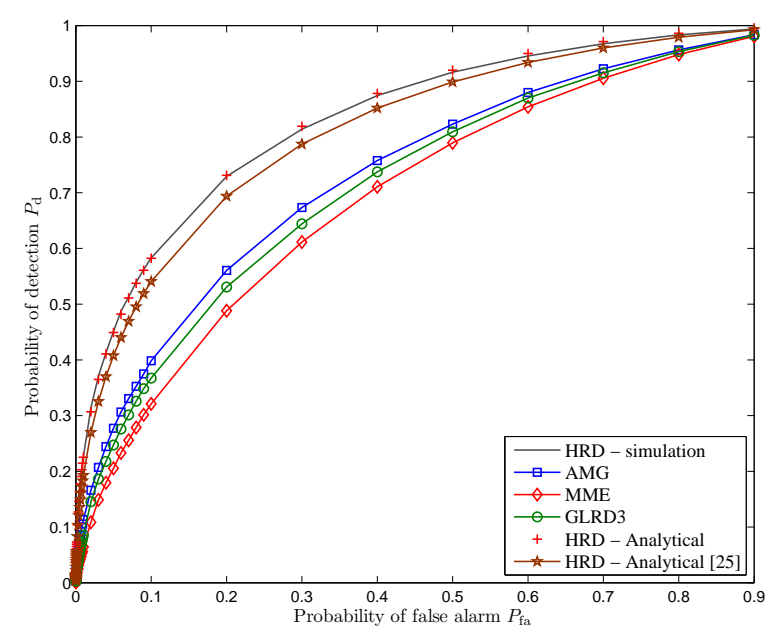

(b)

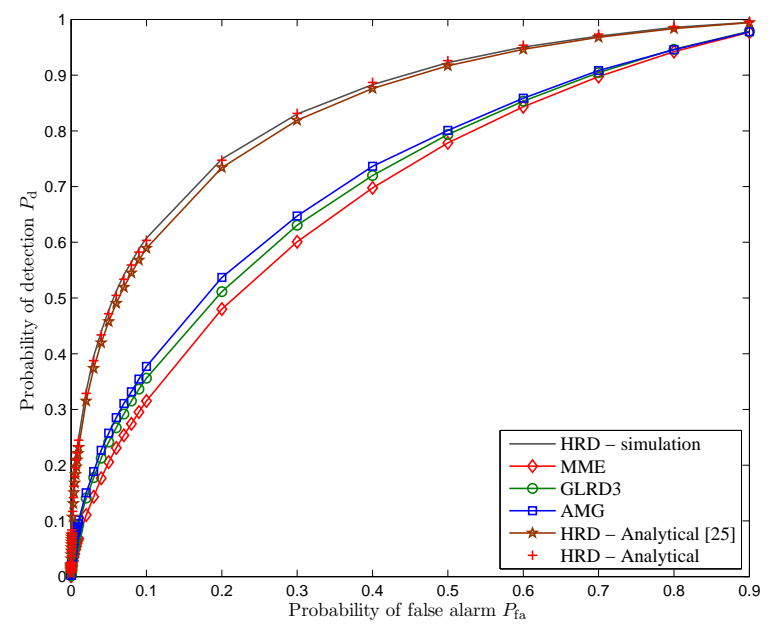

(c)

Fig. 6. The ROC of different detectors at $M=4, K=2$ and: (a) $S N R=0 \mathrm{~dB}, L=30$ (b) $S N R=-1 \mathrm{~dB}, L=50$ (c) $S N R=-2 \mathrm{~dB}, L=100$.

\section{E. Comparison Between the Performance of HRD with the Other Detector}

In Fig. 6, we compare the detection probability of the HRD with other previously reported detectors in terms of their Receiver Operating Characteristics (ROC), as well as our closed-form expression for the performance of the HRD with the approximation in [25]. We assume $M=4$, $K=2$ in all sub-figures, where in different sub-figures we set: (a) $S N R=0 \mathrm{~dB}$ and $L=30$; (b) $S N R=-1 \mathrm{~dB}$ and $L=50$; (c) $S N R=-2 \mathrm{~dB}$ and $L=100$. The PU signal model and the other parameter are the same as Section V-D. Specifically, we compare the GLR Detector-3 (GLRD3) [16, Eqn. (39)], the Arithmetic to Geometric Mean (AMG) [20, Eqn. (14)] detector and the Maximum to Minimum Eigenvalue (MME) [37, Algorithm 1] detector. Fig. 6 shows 
that the HRD outperforms GLR Detector-3, AMG and MME. This is reasonable, since GLR Detector-3, AMG and MME are sensitive to nonuniform per-antenna noise variances, whereas the HRD is robust to that effect. Furthermore, Fig. 6 shows that our closed-form expression for the performance of the HRD is more accurate than the approximation provided in [25] and, moreover, not affected by $L$. In contrast, the accuracy of the approximation in [25] is heavily dependent on $L$ and improves with increasing $L$. Fig. 6 reveals the performance degradation resulting from an inaccurate approximation to the detection and false-alarm probabilities of the HRD, and also, the impact of the new closed-form expressions for those probabilities on the performance improvement of the HRD.

\section{CONCLusion}

We have studied the performance of the HRD for multiantenna spectrum sensing with uncalibrated receivers. Specifically, we have first derived the exact distribution of the HRD statistic under the null hypothesis, which leads to a complicated but closed-form expression for the falsealarm probability. Then, we have derived simpler and tight closed-form expressions for both the false-alarm and detection probabilities by using a moment-based approximation of the HRD distribution under both hypotheses. Finally, simulation are presented to verify the accuracy of the derived results.

\section{APPENDIX A}

\section{PROOF OF LEMMA 1}

To prove that the random variable $\prod_{i=1}^{M} r_{i, i}$ is independent from $T$, we use Basu's theorem.

Basu's theorem. If $W$ is complete and sufficient statistic for the family $\mathcal{P}=\left\{P_{\boldsymbol{\xi}}: \boldsymbol{\xi} \in \boldsymbol{\Xi}\right\}$, then, $W$ is independent from A, for any ancillary statistic A. By definition, a statistic $A$ is ancillary iff its distribution does not depend on $\boldsymbol{\xi}$.

Proof: See [38]

Under hypothesis $\mathcal{H}_{0}$, we have $\boldsymbol{\xi}=\left[\sigma_{1}^{2}, \cdots, \sigma_{M}^{2}\right]^{T}$. Also, the PDF of $\mathbf{Y}$ under $\mathcal{H}_{0}$ is given 
by,

$$
\begin{aligned}
& f\left(\mathbf{Y} \mid \mathcal{H}_{0}, \sigma_{1}^{2}, \cdots, \sigma_{M}^{2}\right)=\frac{\exp \left\{\sum_{l=1}^{L} \mathbf{y}_{l}^{H} \boldsymbol{\Sigma}^{-1} \mathbf{y}_{l}\right\}}{\pi^{M L}|\boldsymbol{\Sigma}|^{L}} \\
& =\frac{\exp \left\{\sum_{l=1}^{L} \sum_{i=1}^{M} \frac{1}{\sigma_{i}^{2}}\left|y_{l, i}\right|^{2}\right\}}{\pi^{M L}|\boldsymbol{\Sigma}|^{L}}=\frac{\exp \left\{\sum_{i=1}^{M} \frac{1}{\sigma_{i}^{2}} r_{i, i}\right\}}{\pi^{M L}|\boldsymbol{\Sigma}|^{L}},
\end{aligned}
$$

where the last equality results from $r_{i, i}=\sum_{l=1}^{L}\left|y_{l, i}\right|^{2}$. From the PDF of $\mathbf{Y}$ under $\mathcal{H}_{0}$, which forms an exponential family, the complete and sufficient statistic is $\left[r_{1,1}, \cdots, r_{M, M}\right]^{T}$. In addition, under $\mathcal{H}_{0}$, the sample covariance matrix $\mathbf{R}$ has an uncorrelated complex Wishart distribution of dimension $M$ and $L$ degrees of freedom, with covariance matrix $\Sigma$, i.e., $\mathbf{R} \sim \mathcal{W}_{C}(M, L, \Sigma)$. By defining $\widetilde{\mathbf{R}} \triangleq \boldsymbol{\Sigma}^{-\frac{1}{2}} \mathbf{R} \boldsymbol{\Sigma}^{-\frac{1}{2}}$ we can rewrite (4) under $\mathcal{H}_{0}$ as,

$$
T=\frac{\prod_{i=1}^{M} \tilde{r}_{i i}}{|\widetilde{\mathbf{R}}|} \gtreqless \mathcal{H}_{1} \mathcal{H}_{0} \eta
$$

where $\widetilde{\mathbf{R}} \sim \mathcal{W}_{C}(M, L, \mathbf{I})$. Thus, it is clear that the distribution of $T$ under $\mathcal{H}_{0}$ does not depend on $\boldsymbol{\xi}=\left[\sigma_{1}^{2}, \cdots, \sigma_{M}^{2}\right]^{T}$. Hence, from Basu's theorem, the random variables $\left\{r_{i, i}\right\}_{i=1}^{M}$ are independent from $T$. In consequence, the random variable $\prod_{i=1}^{M} r_{i, i}$ is also independent from $T$.

\section{APPENDIX B}

\section{Proof OF TheOREM 1}

Under $\mathcal{H}_{1}$, the sample covariance matrix $\mathbf{R}$ is distributed as $\mathbf{R} \sim \mathcal{W}_{C}(M, L, \mathbf{Q})$. Thus, the PDF of the sample covariance matrix $\mathbf{R}$ under $\mathcal{H}_{1}$ is given by [39],

$$
f_{\mathbf{R}}(\mathbf{R})=\frac{|\mathbf{R}|^{L-M} \operatorname{etr}\left(-\mathbf{Q}^{-1} \mathbf{R}\right)}{\Gamma_{\mathrm{M}}(L)|\mathbf{Q}|^{L}} .
$$

Consequently, the $m^{\text {th }}$ moment of $T$, where $m \in \mathbb{N}$, under $\mathcal{H}_{1}$ can be computed as: $\operatorname{E}\left\{T^{m} \mid \mathcal{H}_{1}\right\}=$

$$
\begin{aligned}
& =\int_{\mathbf{R} \succ 0}\left(\frac{\prod_{i=1}^{M} r_{i, i}}{|\mathbf{R}|}\right)^{m} \frac{|\mathbf{R}|^{L-M} \operatorname{etr}\left(-\mathbf{Q}^{-1} \mathbf{R}\right)}{\Gamma_{\mathrm{M}}(L)|\mathbf{Q}|^{L}} \mathrm{~d} \mathbf{R} \\
& =\frac{\Gamma_{\mathrm{M}}(L-m)}{\Gamma_{\mathrm{M}}(L)|\mathbf{Q}|^{m}} \int_{\mathbf{R} \succ 0}\left(\prod_{i=1}^{M} r_{i, i}\right)^{m} \frac{|\mathbf{R}|^{L-M-m} \operatorname{etr}\left(-\mathbf{Q}^{-1} \mathbf{R}\right)}{\Gamma_{\mathrm{M}}(L-m)|\mathbf{Q}|^{L-m}} \mathrm{~d} \mathbf{R} \\
& =\frac{\Gamma_{\mathrm{M}}(L-m)}{\Gamma_{\mathrm{M}}(L)|\mathbf{Q}|^{m}} \mathrm{E}\left\{\left(\prod_{i=1}^{M} r_{i, i}^{\prime}\right)^{m}\right\},
\end{aligned}
$$

where $\mathbf{R}^{\prime} \sim \mathcal{W}_{C}(M, L-m, \mathbf{Q})$. Thus, to find the $m^{\text {th }}$ moment of $T$ under $\mathcal{H}_{1}$, we should obtain the correlation between the random variables $r_{i, i}^{\prime m}$. In that, we use the Characteristic Function (CF) 
of $\mathbf{R}^{\prime}$. Let $\Theta$ be a $M \times M$ Hermitian matrix; then, the CF of the random variables $r_{1,1}^{\prime}, \cdots, r_{M, M}^{\prime}$, $2 \Re\left\{r_{1,2}^{\prime}\right\}, 2 \Im\left\{r_{1,2}^{\prime}\right\}, \cdots, 2 \Re\left\{r_{M-1, M}^{\prime}\right\}, 2 \Im\left\{r_{M-1, M}^{\prime}\right\}$ reads,

$$
\phi(\Theta)=\mathrm{E}\left\{\operatorname{etr}\left(\mathrm{j} \mathbf{R}^{\prime} \Theta\right)\right\}=\left|\mathbf{I}_{M}-\mathrm{j} \Theta \mathbf{Q}\right|^{-(L-m)} .
$$

and the correlation between all the terms $r_{i, i}^{\prime m}$ is obtained as,

$$
\mathrm{E}\left\{\left(\prod_{i=1}^{M} r_{i, i}^{\prime}\right)^{m}\right\}=\left.\mathrm{j}^{-m M} \frac{\partial^{m M} \phi(\boldsymbol{\Theta})}{\partial \theta_{1,1}^{m} \cdots \partial \theta_{M, M}^{m}}\right|_{\boldsymbol{\Theta}=\mathbf{0}} .
$$

Thus, we employ the following Lemma to compute (50).

Lemma 3. Let

$$
\left.D_{\boldsymbol{u}, N}(\boldsymbol{Q}) \doteq \frac{\partial^{n}\left|\mathbf{I}_{M}-\mathrm{j} \Theta \boldsymbol{Q}\right|^{-N}}{\partial \theta_{1,1}^{u_{1}} \cdots \partial \theta_{M, M}^{u_{M}}}\right|_{\boldsymbol{\Theta = 0}},
$$

with $\boldsymbol{u} \doteq\left[u_{1}, \cdots, u_{M}\right]^{T} \in \mathbb{N}^{M}$ and $n \doteq \sum_{i=1}^{M} u_{i}$. The generating function for all derivatives $D_{\boldsymbol{u}, N}(\boldsymbol{Q})$ of order $n$ is given by the following multivariate homogeneous polynomial of degree $n$,

$$
\begin{aligned}
\mathcal{A}_{n}(\boldsymbol{X} ; \boldsymbol{Q}) & =\mathrm{j}^{n} \sum_{k=1}^{n} N^{k} \sum_{\mathcal{W}(n, k)} \frac{1}{\prod_{i=1}^{d} w_{i} !} \prod_{i=1}^{d}\left(\frac{\operatorname{tr}\left[(\boldsymbol{X} \boldsymbol{Q})^{i}\right]}{i}\right)^{m_{i}} \\
& =\mathrm{j}^{n} \sum_{l_{1}+l_{2}+\cdots+l_{M}=n} c(\boldsymbol{l}, n) \cdot x_{1}^{l_{1}} x_{2}^{l_{2}} \cdots x_{M}^{l_{M}}
\end{aligned}
$$

with $\boldsymbol{l} \doteq\left[l_{1}, l_{2}, \cdots, l_{M}\right]^{T} \in \mathbb{N}^{M}, d \doteq n-k+1, \boldsymbol{X} \doteq \operatorname{diag}\{\boldsymbol{\Theta}\}^{7}$ and where $\mathcal{W}(n, k)$ denotes the set of d-tuples $\left(w_{1}, \cdots, w_{d}\right)$ that fulfil the following constraints,

$$
\left\{\begin{array}{l}
\sum_{i=1}^{d} w_{i}=k, \\
\sum_{i=1}^{d} i \cdot w_{i}=n,
\end{array} \quad, \quad m_{i} \geq 0 .\right.
$$

Accordingly, we will have,

$$
D_{\boldsymbol{u}, N}(\boldsymbol{Q})=j^{n}\left(\prod_{i=i}^{M} u_{i} !\right) c(\boldsymbol{u}, n) .
$$

Proof:

Stage 1: Let $\Theta=\boldsymbol{X}+\Theta_{0}$, where $\Theta_{0}$ has zeroes along its diagonal and its off-diagonal entries

\footnotetext{
${ }^{7}$ For the sake of notational simplicity, the diagonal entries of $\boldsymbol{X}$, i.e. $x_{i, i}$, are referred to by $x_{i}$.
} 
equal those of $\Theta$. To compute the multiple derivative of $\left|\mathbf{I}_{M}-\mathrm{j} \Theta Q\right|^{-N}$ with respect to the diagonal entries of $\Theta$, or equivalently, $\boldsymbol{X}$, we have,

$$
\begin{aligned}
\left.\frac{\partial^{n}\left|\mathbf{I}_{M}-\mathrm{j} \boldsymbol{\Theta} \boldsymbol{Q}\right|^{-N}}{\partial \theta_{1,1}^{u_{1}} \cdots \partial \theta_{M, M}^{u_{M}}}\right|_{\boldsymbol{\Theta =}=\mathbf{0}} & =\left.\frac{\partial^{n}\left|\left(\mathbf{I}-\mathbf{j} \boldsymbol{\Theta}_{0} \boldsymbol{Q}\right)-\mathbf{j} \boldsymbol{X} \boldsymbol{Q}\right|^{-N}}{\partial x_{1}^{u_{1}} \cdots \partial x_{M}^{u_{M}}}\right|_{\boldsymbol{\Theta}=\mathbf{0}} \\
& =\left.\frac{\partial^{n}|\mathbf{I}-\mathbf{j} \boldsymbol{X} \boldsymbol{Q}|^{-N}}{\partial x_{1}^{u_{1}} \cdots \partial x_{M}^{u_{M}}}\right|_{\boldsymbol{X}=\mathbf{0}} .
\end{aligned}
$$

Now, we can write,

$$
|\mathbf{I}-\mathrm{j} \boldsymbol{X} \boldsymbol{Q}|^{-N}=\exp (-N \log |\mathbf{I}-\mathrm{j} \boldsymbol{X} \boldsymbol{Q}|)
$$

We should note that $|\mathbf{I}-\mathrm{j} \boldsymbol{X} \boldsymbol{Q}|$ is generally a complex number. Nonetheless, as we are solely interested in a neighborhood of $\boldsymbol{X} \sim \boldsymbol{0}$ for computing the derivatives, we can safely take the principal branch of the logarithm function. Thus, we can exploit the following power series [40, pp. 1029],

$$
\log |\mathbf{I}+\boldsymbol{\Upsilon}|=\sum_{n=1}^{\infty} \frac{(-1)^{n+1}}{n} \operatorname{tr}\left[\Upsilon^{n}\right]
$$

So, setting $\Upsilon=-\mathrm{j} \boldsymbol{X} \boldsymbol{Q}, \tau=\operatorname{tr}[\mathbf{\Upsilon}]$ and $\tilde{\boldsymbol{\Upsilon}}=\tau^{-1} \Upsilon \mathbf{\Upsilon}$, we get,

$$
|\mathbf{I}-\mathrm{j} \boldsymbol{X} \boldsymbol{Q}|^{-N}=\exp \left(\sum_{n=1}^{\infty} \frac{N(-1)^{n} \operatorname{tr}\left[\tilde{\Upsilon}^{n}\right](n-1) !}{n !} \tau^{n}\right) \text {. }
$$

Stage 2: We resort to the following formal power series,

$$
\exp \left(\sum_{n=1}^{\infty} \frac{\vartheta_{n}}{n !} \tau^{n}\right)=\sum_{n=0}^{\infty} \frac{B_{n}\left(\vartheta_{1}, \cdots, \vartheta_{n}\right)}{n !} \tau^{n},
$$

where $B_{n}\left(\vartheta_{1}, \cdots, \vartheta_{n}\right)$ constitutes the complete Bell polynomials [41]. The complete Bell polynomials are expressed from the partial Bell polynomials $B_{n, k}\left(\vartheta_{1}, \cdots, \vartheta_{n-k+1}\right)$ as [41],

$$
B_{n}\left(\vartheta_{1}, \cdots, \vartheta_{n}\right)=\sum_{k=1}^{n} B_{n, k}\left(\vartheta_{1}, \cdots, \vartheta_{n-k+1}\right)
$$

where,

$$
B_{n, k}\left(\vartheta_{1}, \cdots, \vartheta_{n-k+1}\right) \doteq \sum_{\mathcal{W}(n, k)} \frac{n !}{\prod_{i=1}^{n-k+1} w_{i} !} \prod_{i=1}^{n-k+1}\left(\frac{\vartheta_{i}}{i !}\right)^{w_{i}}
$$

with $\mathcal{W}(n, k)$ the set of $d$-tuples $\left(w_{1}, \cdots, w_{d}\right)$ defined in (54).

Stage 3: We apply (60) to (59). From (60) and (59), we set,

$$
\vartheta_{i}=N(-1)^{i} \operatorname{tr}\left[\tilde{\Upsilon}^{i}\right](i-1) !
$$


From (60) and the previous definition of the $\vartheta_{i}^{\prime}$ 's, we have,

$$
|\mathbf{I}-\mathrm{j} \boldsymbol{X} \boldsymbol{Q}|^{-N}=\sum_{n=0}^{\infty} \frac{B_{n}\left(-N \operatorname{tr}[\tilde{\boldsymbol{\Upsilon}}], \cdots, N(-1)^{n} \operatorname{tr}\left[\tilde{\boldsymbol{\Upsilon}}^{n}\right](n-1) !\right)}{n !} \tau^{n}
$$

From the definition of the complete Bell polynomials in (61) and (62), we have,

$$
\begin{aligned}
|\mathbf{I}-\mathrm{j} \boldsymbol{X} \boldsymbol{Q}|^{-N} & =\sum_{n=0}^{\infty} \sum_{k=1}^{n} \frac{B_{n, k}\left(-N \operatorname{tr}[\tilde{\boldsymbol{\Upsilon}}], \cdots, N(-1)^{n-k+1} \operatorname{tr}\left[\tilde{\boldsymbol{\Upsilon}}^{n-k+1}\right](n-k) !\right)}{n !} \tau^{n} \\
& =\sum_{n=0}^{\infty} \sum_{k=1}^{n} \sum_{\mathcal{W}(n, k)} \frac{N^{k}(-1)^{n}}{\prod_{i=1}^{n-k+1} w_{i} !} \prod_{i=1}^{n-k+1}\left(\frac{\operatorname{tr}\left[\tilde{\boldsymbol{\Upsilon}}^{i}\right]}{i}\right)^{w_{i}} \tau^{n} .
\end{aligned}
$$

According to $\Upsilon=\tau \tilde{\Upsilon}$, we find,

$$
|\mathbf{I}-\mathbf{j} \boldsymbol{X} \boldsymbol{Q}|^{-N}=\sum_{n=0}^{\infty} \sum_{k=1}^{n} \sum_{\mathcal{W}(n, k)} \frac{N^{k}(-1)^{n}}{\prod_{i=1}^{n-k+1} w_{i} !} \prod_{i=1}^{n-k+1}\left(\frac{\operatorname{tr}\left[\boldsymbol{\Upsilon}^{i}\right]}{i}\right)^{w_{i}} .
$$

Then, by substituting $\Upsilon=-\mathrm{j} \boldsymbol{X} \boldsymbol{Q}$, we get,

$$
\begin{aligned}
|\mathbf{I}-\mathrm{j} \boldsymbol{X} \boldsymbol{Q}|^{-N} & =\sum_{n=0}^{\infty} \mathrm{j}^{n} \sum_{k=1}^{n} N^{k} \sum_{\mathcal{W}(n, k)} \frac{1}{\prod_{i=1}^{n-k+1} w_{i} !} \prod_{i=1}^{n-k+1}\left(\frac{\operatorname{tr}\left[(\boldsymbol{X} \boldsymbol{Q})^{i}\right]}{i}\right)^{w_{i}} \\
& =\sum_{n=0}^{\infty} \mathcal{A}_{n}(\boldsymbol{X} ; \boldsymbol{Q}) .
\end{aligned}
$$

Due to the constraint $\sum_{i=1}^{n-k+1} i \cdot w_{i}=n$ in the definition of $\mathcal{W}(n, k), \mathcal{A}_{n}(\boldsymbol{X} ; \boldsymbol{Q})$ is an homogeneous polynomial of degree $n$ in the components of the diagonal matrix $\boldsymbol{X}$, that is,

$$
\mathcal{A}_{n}(\boldsymbol{X} ; \boldsymbol{Q})=\mathrm{j}^{n} \sum_{l_{1}+\cdots+l_{M}=n} c(\boldsymbol{l}, n) \cdot x_{1}^{l_{1}} x_{2}^{l_{2}} \cdots x_{M}^{l_{M}}
$$

Thus, we can say that $\mathcal{A}_{n}(\boldsymbol{X} ; \boldsymbol{Q})$ is the generating function for all derivatives $D_{\boldsymbol{u}, N}(\boldsymbol{Q})$ of order $n$, so that,

$$
\begin{aligned}
D_{\boldsymbol{u}, N}(\boldsymbol{Q}) & =\left.\frac{\partial^{n}|\mathbf{I}-\mathrm{j} \boldsymbol{X} \boldsymbol{Q}|^{-N}}{\partial x_{1}^{u_{1}} \cdots \partial x_{M}^{u_{M}}}\right|_{\boldsymbol{X}=\mathbf{0}}=\left.\frac{\partial^{n} \mathcal{A}_{n}(\boldsymbol{X} ; \boldsymbol{Q})}{\partial x_{1}^{u_{1}} \cdots \partial x_{M}^{u_{M}}}\right|_{\mathbf{X}=\mathbf{0}} \\
& =j^{n}\left(\prod_{i=i}^{M} u_{i} !\right) c(\boldsymbol{u}, n) .
\end{aligned}
$$

Note that we have transformed the computation of the multiple derivative of the inverse of a multivariate polynomial in (51) to the much less complex computation of the derivative of a plain multivariate polynomial expressed in terms of traces (Lemma 3). Thus, we can now use more straightforward methods to compute the multiple derivative in (51), that is, (50). In 
fact, we can obtain $c(\boldsymbol{u}, n)$ by extracting the corresponding coefficients of $x_{1}^{u_{1}} \cdots x_{M}^{u_{M}}$ from the multivariate homogeneous polynomial $\mathcal{A}_{n}(\boldsymbol{X} ; \boldsymbol{Q})$ and then compute $D_{\boldsymbol{u}, N}(\boldsymbol{Q})$ by using (55). Hence, we continue by expanding $\mathcal{A}_{n}(\boldsymbol{X} ; \boldsymbol{Q})$.

Let us define $\Phi_{n, k}$ as,

$$
\Phi_{n, k} \doteq \sum_{\mathcal{W}(n, k)} \frac{1}{\prod_{i=1}^{d} w_{i} !} \prod_{i=1}^{d}\left(\frac{\operatorname{tr}\left[(\boldsymbol{X} \boldsymbol{Q})^{i}\right]}{i}\right)^{w_{i}} .
$$

Then, using the property of matrix trace operation,

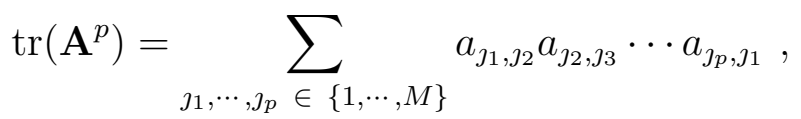

in which $\mathbf{A}$ is a $M \times M$ matrix, we could expand $\Phi_{n, k}$ as,

$$
\begin{aligned}
& \Phi_{n, k}=\sum_{\mathcal{W}(n, k)} \frac{1}{\prod_{i=1}^{d} w_{i} !}\left(\sum_{\jmath_{p} \in\{1, \cdots, M\}} q_{\jmath_{p}, \jmath_{p}} x_{\jmath_{p}}\right)^{w_{1}} \\
& \times \prod_{i=2}^{d}\left(\sum_{\jmath_{1}, \cdots, \jmath_{i} \in\{1, \cdots, M\}} \frac{q_{\jmath_{1}, \jmath_{2}} q_{\jmath_{2}, \jmath_{3}} \cdots q_{\jmath_{i}, \jmath_{1}}}{i} \prod_{p=1}^{i} x_{\jmath_{p}}\right)^{w_{i}} .
\end{aligned}
$$

A product of sums can be expressed as a sum of products as multiplication distributes over addition. We could obtain the expansion of (72) by repeatedly replacing subexpressions in (72) by the equivalent sum of products, continuing until the expression becomes a sum of products as shown in (73),

According to Lemma 3, $c(\boldsymbol{u}, n)$ equals the coefficient of $x_{1}^{u_{1}} \cdots x_{M}^{u_{M}}$ in (52). Thus, w.r.t. (73), $c(\boldsymbol{u}, n)$ can be extracted as (74), with $G$ defined as the set of all multiset permutations of the multiset $\left(A=\{1, \cdots, M\},\left\{f(i)=u_{i}: i \in A\right\}\right)$ for which $A$ is the underlying set of elements and $f(i)$ the multiplicity function. The term $\aleph$ in (74) can be rewritten as,

$$
\prod_{p=1}^{n} q_{\jmath_{p}, \jmath_{\pi(p)}}
$$

with $\pi$ a permutation of the symmetric group $\mathcal{S}_{n}$ on the set $\{1,2, \cdots, n\}$ with portrait ${ }^{8} \ell=$ $\left(\left(w_{1}, w_{2}, \cdots, w_{d}\right),\left(\mathbf{0}_{k-1}\right)\right)$ and the set of cycles $\mathcal{D}(\pi)$ which is implicity defined from (74).

\footnotetext{
${ }^{8}$ To each $\pi$ in $\mathcal{S}_{n}$ we associate the $n$-tuple $\ell=\left(c_{1}, c_{2}, \cdots, c_{n}\right)$ where $c_{\jmath}$ is the number of cycles of length $\jmath$ in $\pi$. Such a $n$-tuple $\ell$ is called the portrait of $\pi$. Note that different permutations in $\mathcal{S}_{n}$ may have an equal portrait.
} 


$$
\begin{aligned}
& \Phi_{n, k} \\
& =\sum_{\mathcal{W}(n, k)} \frac{1}{\prod_{i=1}^{d} w_{i} !}\left(\sum_{\jmath_{1}, \cdots, \jmath_{w_{1}} \in\{1, \cdots, M\}} \prod_{p=1}^{w_{1}} q_{\jmath_{p}, \jmath_{p}} x_{\jmath_{p}}\right) \\
& \times \prod_{i=2}^{d} \sum_{\jmath_{1}, \cdots, \jmath_{i . w_{i}} \in\{1, \cdots, M\}} \frac{\prod_{p=1}^{i \cdot w_{i}} x_{\jmath_{p}}}{i^{w_{i}}} \prod_{l=1}^{w_{i}} q_{\jmath_{(l-1) i+1}, \jmath_{(l-1) i+2}} q_{\jmath_{(l-1) i+2}, \jmath_{(l-1) i+3}} \cdots q_{\jmath_{l i}, \jmath_{(l-1) i+1}}
\end{aligned}
$$

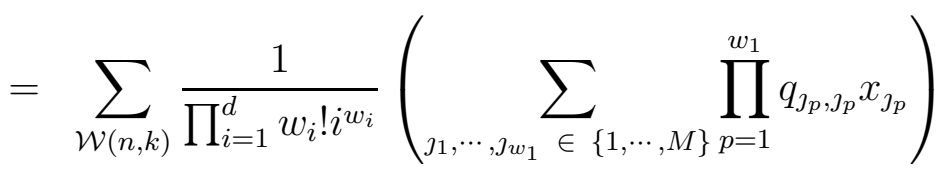

$$
\begin{aligned}
& \times\left(\sum_{\jmath_{1}, \cdots, \jmath_{\sum_{i=2}^{d}}^{d}, w_{i}} \in\left\{\prod_{p=1}^{\sum_{i=2}^{d} i \cdot \cdots, w_{i}} x_{\jmath_{p}} \prod_{i=2}^{d} \prod_{l=1}^{w_{i}} q_{\jmath_{(l-1) i+1}, \jmath_{(l-1) i+2}} q_{\jmath_{(l-1) i+2}, \jmath_{(l-1) i+3}} \cdots q_{\jmath_{l i}, \jmath_{(l-1) i+1}}\right)\right. \\
& =\sum_{\mathcal{W}(n, k)} \frac{1}{\prod_{i=1}^{d} w_{i} ! i^{w_{i}}} \sum_{\jmath_{1}, \cdots, \jmath_{n}} \prod_{\{1, \cdots, M\}}^{n} x_{p=1} x_{\jmath_{p}} \prod_{p=1}^{w_{1}} q_{\jmath_{p}, \jmath_{p}} \\
& \times \prod_{i=2}^{d} \prod_{l=1}^{w_{i}} q_{\jmath_{w_{1}+(l-1) i+1}, \jmath_{w_{1}+(l-1) i+2}} q_{\jmath_{w_{1}+(l-1) i+2}, \jmath_{w_{1}+(l-1) i+3}} \cdots q_{\jmath_{w_{1}+l i}, \jmath_{w_{1}+(l-1) i+1}} .
\end{aligned}
$$

$$
\begin{aligned}
& c(\boldsymbol{u}, n)=\sum_{k=1}^{n} N^{k} \sum_{\mathcal{W}(n, k)} \frac{1}{\prod_{i=1}^{d} w_{i} ! i^{w_{i}}} \sum_{\left(\jmath_{1}, \cdots, \jmath_{n}\right) \in G} \\
& \overbrace{\prod_{p=1}^{w_{1}} q_{\jmath_{p}, \jmath_{p}} \prod_{i=2}^{d} \prod_{l=1}^{w_{i}} q_{\jmath_{w_{1}+(l-1) i+1}, \jmath_{w_{1}+(l-1) i+2}} q_{\jmath_{w_{1}+(l-1) i+2}, \jmath_{w_{1}+(l-1) i+3}} \cdots q_{\jmath_{w_{1}+l i}, \jmath_{w_{1}+(l-1) i+1}}}^{N} .
\end{aligned}
$$

Defining the $n$-tuple $\left(i_{1}, i_{2}, \cdots, i_{n}\right) \doteq\left(\left(\mathbf{1}_{u_{1}}\right),\left(\mathbf{2}_{u_{2}}\right), \cdots,\left(\mathbf{M}_{u_{M}}\right)\right)$, it is easy to verify that

$$
\sum_{\left(\jmath_{1}, \cdots, \jmath_{n}\right) \in G} \prod_{p=1}^{n} q_{\jmath_{p}, \jmath_{\pi(p)}}=\frac{\prod_{i=1}^{d} w_{i} ! i^{w_{i}}}{\prod_{i=1}^{M} u_{i} !} \sum_{\pi \in \mathcal{G}} \prod_{p=1}^{n} q_{i_{p}, i_{\pi(p)}} .
$$

with $\mathcal{G}$ defined as the subgroup of the symmetric group $\mathcal{S}_{n}$ including all permutations with portrait $\boldsymbol{\ell}=\left(\left(w_{1}, w_{2}, \cdots, w_{d}\right),\left(\mathbf{0}_{k-1}\right)\right)$. By substituting (76) in (74),

$$
c(\boldsymbol{u}, n)=\sum_{k=1}^{n} \frac{N^{k}}{\prod_{i=1}^{M} u_{i} !} \sum_{\mathcal{W}(n, k)} \sum_{\pi \in \mathcal{G}} \prod_{p=1}^{n} q_{i_{p}, i_{\pi(p)}} .
$$


The summations over $\mathcal{W}(n, k)$ and $\pi \in \mathcal{G}$ in (77) could be merged into a summation over all the permutations $\pi$ which have a portrait $\boldsymbol{\ell}=\left(\left(w_{1}, w_{2}, \cdots, w_{d}\right),\left(\mathbf{0}_{k-1}\right)\right)$ such that the $d$-tuples $\left(w_{1}, w_{2}, \cdots, w_{d}\right)$ fulfil the constraints (54).

Lemma 4. If $\mathcal{I}_{n}$ is the subgroup of $\mathcal{S}_{n}$ including all the permutations $\pi$ which have a portrait $\boldsymbol{\ell}=\left(\left(w_{1}, w_{2}, \cdots, w_{d}\right),\left(\mathbf{0}_{k-1}\right)\right)$ such that the d-tuples $\left(w_{1}, w_{2}, \cdots, w_{d}\right)$ fulfil the constraints (54), then we could equivalently say that $\mathcal{I}_{n}$ is the subgroup of $\mathcal{S}_{n}$ including all the permutations $\pi$ which are permutated to the identity permutation only with $(n-k)$ permutations.

Proof: It is easy to verify that every $\pi \in \mathcal{I}_{n}$ needs $\sum_{i=1}^{d}(i-1) w_{i}$ permutations to be permutated to the identity permutation. On the other hand, from the constraints (54): $\sum_{i=1}^{d}(i-$ 1) $w_{i}=n-k$, and thus, the proof is complete.

Now by using Lemma 4, (77) can be rewritten as,

$$
c(\boldsymbol{u}, n)=\sum_{k=1}^{n} \frac{N^{k}}{\prod_{i=1}^{M} u_{i} !} \sum_{\pi \in \mathcal{I}_{n}} \prod_{p=1}^{n} q_{i_{p}, i_{\pi(p)}},
$$

where $\mathcal{I}_{n}$ is the subgroup of $\mathcal{S}_{n}$ including all the permutations $\pi$ which are permutated to the identity permutation only with $(n-k)$ permutations. From (55), we have,

$$
D_{\boldsymbol{u}, N}(\boldsymbol{Q})=j^{n} \sum_{k=1}^{n} N^{k} \psi_{n, k}
$$

where $\psi_{n, k}=\sum_{\pi \in \mathcal{I}_{n}} \prod_{p=1}^{n} q_{i_{p}, i_{\pi(p)}}$. Now, by setting $\mathbf{u}=m \times \mathbf{1}_{M}$ and $N=L-m$ in (79) and substituting the result in (50), we have,

$$
\mathrm{E}\left\{\left(\prod_{i=1}^{M} r_{i, i}^{\prime}\right)^{m}\right\}=\sum_{k=1}^{m M}(L-m)^{k} \psi_{m M, k}
$$

Finally, substituting (80) in (48), yields Theorem 1.

\section{APPENDIX C}

\section{APPLICATION OF THEOREM 1}

To obtain $\psi_{M, M}, \psi_{2 M, 2 M}, \psi_{M, M-1}$ and $\psi_{2 M, 2 M-1}$, we express each permutation as a product of cycles corresponding to the orbits of the permutation. For instance: the permutation $(2,1,4,3,5)$ of the set $\{1,2,3,4,5\}$ is denoted $(12)(34)(5)$.

1) $\psi_{m M, m M}$ : the subgroup $\mathcal{I}_{m M}$ includes only the identity permutation $\left(i_{1}\right)\left(i_{2}\right) \cdots\left(i_{m M}\right)$. As $\left(i_{1}, i_{2}, \cdots, i_{m M}\right)=\left(\left(\mathbf{1}_{m}\right),\left(\mathbf{2}_{m}\right), \cdots,\left(\mathbf{M}_{m}\right)\right)$ and using $\mathbf{D}_{Q}^{2}=\operatorname{diag}[\mathbf{Q}]$, we get: $\psi_{m M, m M}=$ $\prod_{i=1}^{M} q_{i, i}^{m}=\left|\mathbf{D}_{Q}^{2}\right|^{m}$. 
2) $\psi_{M, M-1}$ : the subgroup $\mathcal{I}_{M}$ includes the permutations $\left\{\left(i_{k} i_{p}\right) \prod_{j=1, j \neq k, p}^{M}\left(i_{j}\right)\right\}_{1 \leq k<p \leq M}$. As $\left(i_{1}, i_{2}, \cdots, i_{M}\right)=(1,2, \cdots, M)$ and using $\mathbf{C}_{Q}=\mathbf{D}_{Q}^{-1} \mathbf{Q D}_{Q}^{-1}$, we get,

$$
\begin{aligned}
\psi_{M, M-1} & =\sum_{i>k} q_{k, i} q_{i, k} \prod_{j=1, j \neq i, k}^{M} q_{j, j} \\
& =\left(\prod_{j=1}^{M} q_{j, j}\right) \sum_{i>k} \frac{\left|q_{i, k}\right|^{2}}{q_{i, i} q_{k, k}}=\left|\mathbf{D}_{Q}^{2}\right| \frac{1}{2} \sum_{i \neq k} \frac{\left|q_{i, k}\right|^{2}}{q_{i, i} q_{k, k}} \\
& =\frac{1}{2}\left|\mathbf{D}_{Q}^{2}\right| \cdot\left\|\mathbf{C}_{Q}-\mathbf{I}_{M}\right\|_{F}^{2}
\end{aligned}
$$

3) $\psi_{2 M, 2 M-1}$ : the subgroup $\mathcal{I}_{2 M}$ includes the permutations $\left\{\left(i_{k} i_{p}\right) \prod_{j=1, j \neq k, p}^{2 M}\left(i_{j}\right)\right\}_{1 \leq k<p \leq 2 M}$. Since $\left(i_{1}, i_{2}, \cdots, i_{2 M}\right)=\left(\left(\mathbf{1}_{2}\right),\left(\mathbf{2}_{2}\right), \cdots,\left(\mathbf{M}_{2}\right)\right)$, we get,

$$
\begin{aligned}
\psi_{2 M, 2 M-1} & =M \prod_{i=1}^{M} q_{i, i}^{2}+4 \sum_{i>k} q_{k, i} q_{i, k} q_{i, i} q_{k, k} \prod_{j=1, j \neq k, i}^{M} q_{j, j}^{2} \\
& =\left(\prod_{j=1}^{M} q_{j, j}^{2}\right)\left(M+4 \sum_{i>k} \frac{\left|q_{k, i}\right|^{2}}{q_{i, i} q_{k, k}}\right) \\
& =\left|\mathbf{D}_{Q}^{2}\right|^{2}\left(M+2 \sum_{i \neq k} \frac{\left|q_{k, i}\right|^{2}}{q_{i, i} q_{k, k}}\right) \\
& =\left|\mathbf{D}_{Q}^{2}\right|^{2}\left(M+2\left\|\mathbf{C}_{Q}-\mathbf{I}_{M}\right\|_{F}^{2}\right)
\end{aligned}
$$

Hence, substituting the previous coefficients into (34), we get the two (approximate) first moments in (40) and (41).

\section{APPENDIX D}

\section{SOME LARGE- $L$ APPROXIMATIONS UNDER $\mathcal{H}_{0}$ AND $\mathcal{H}_{1}$}

We start developing $\mu_{T \mid \mathcal{H}_{1}}^{2}$ in (40) up to $1 / L^{2}$. From (40), let $\Delta \doteq\left\|\mathbf{C}_{Q}-\mathbf{I}_{M}\right\|_{F}^{2}$ and $\gamma_{1} \doteq$ $\frac{1}{\left|\mathbf{C}_{Q}\right|^{2}}\left(1+\frac{a_{11}}{L-1}+\frac{a_{12}}{(L-1)^{2}}\right)^{2}$ be defined, with $a_{11}=\frac{1}{2} \Delta$ and $a_{12}=\psi_{M, M-2}$ (not calculated in this paper), so that $\ln \mu_{T \mid \mathcal{H}_{1}}^{2}=A+\ln \gamma_{1}$, where $A \doteq \ln \mu_{T \mid \mathcal{H}_{0}}^{2}$ (see (21)). So, using the small- $x$ approximation $\ln (1+x) \simeq x-\frac{1}{2} x^{2}$ on $A=2 \sum_{j=1}^{M} \ln \frac{1-1 / L}{1-j / L}$,

$$
A \simeq 2 \sum_{j=1}^{M} \frac{j-1}{L}+\sum_{j=1}^{M} \frac{j^{2}-1}{L^{2}} .
$$

Thus, using $\sum_{j=1}^{M} j=\frac{M(M+1)}{2}$ and $\sum_{j=1}^{M} j^{2}=\frac{M(M+1)(2 M+1)}{6}$, we have, after some algebra,

$$
A=\frac{M(M-1)}{L}+\frac{M(M-1)(2 M+5)}{6 L^{2}} .
$$


Using the second-order approximation to $\ln (1+x)$, we also get, up to terms in $1 /(L-1)^{2}$ : $\ln \gamma_{1} \simeq \ln \frac{1}{\left|\mathbf{C}_{Q}\right|^{2}}+\frac{2 a_{11}}{L-1}+\frac{2 a_{12}+a_{11}}{(L-1)^{2}}$. As $\frac{1}{L-1} \simeq \frac{1}{L}\left(1+\frac{1}{L}+\frac{1}{L^{2}}\right)$ and $\frac{1}{(L-1)^{2}} \simeq \frac{1}{L^{2}}\left(1+\frac{2}{L}+\frac{3}{L^{2}}\right)$, we finally obtain, up to $1 / L^{2}$,

$$
\begin{aligned}
\ln \mu_{T \mid \mathcal{H}_{1}}^{2} & \simeq \frac{M(M-1)}{L}\left(1+\frac{2 M+5}{6 L}\right)+\ln \frac{1}{\left|\mathbf{C}_{Q}\right|^{2}} \\
& +\frac{2 a_{11}}{L}+\frac{2 a_{11}+2 a_{12}+a_{11}^{2}}{L^{2}}
\end{aligned}
$$

We approach now the second moment in (41) up to $1 / L^{2}$. Defining $\gamma_{2} \doteq \frac{1}{\left|\mathbf{C}_{Q}\right|^{2}}\left(1+\frac{a_{21}}{L-2}+\frac{a_{22}}{(L-2)^{2}}\right)$, with $a_{21}=M+2 \Delta$ and $a_{22}=\psi_{2 M, 2 M-2}$ (not calculated in this paper), we have $\ln \mu_{2, T \mid \mathcal{H}_{1}}=$ $B+C+\ln \gamma_{2}$, where we define $B \doteq 2 \sum_{j=1}^{M} \ln \frac{L-2}{L-j}$, with,

$$
\begin{aligned}
B & \simeq 2 \sum_{j=1}^{M} \frac{j-2}{L}+\sum_{j=1}^{M} \frac{j^{2}-4}{L^{2}}=\frac{(M-1)(M-2)-2}{L} \\
& +\frac{1}{L^{2}}\left(\frac{M(M+1)(2 M+1)}{6}-4 M\right)
\end{aligned}
$$

and where we define $C \doteq \ln \frac{L-1}{L-M-1}$, with,

$$
C \simeq \frac{M}{L}+\frac{M^{2}+2 M}{2 L^{2}}+\frac{(M+1)^{3}-1}{3 L^{3}}
$$

Back to $\mathcal{H}_{0}$, for computing the approximation to $\ln \mu_{2, T \mid \mathcal{H}_{0}}$ we also need to define $D \doteq M \ln \frac{L-2}{L-1}$, so that,

$$
D \simeq-\frac{M}{L}-\frac{\frac{3}{2} M}{L^{2}}-\frac{\frac{7}{3} M}{L^{3}}
$$

Therefore, we get the following approximation,

$$
\begin{aligned}
& \ln \mu_{2, T \mid \mathcal{H}_{0}}-\ln \mu_{T \mid \mathcal{H}_{0}}^{2} \simeq C+D \\
& \simeq \frac{M(M-1)}{2 L^{2}}+\frac{M(M-1)(M+4)}{3 L^{3}} .
\end{aligned}
$$

Picking up for the second moment under $\mathcal{H}_{1}$ : we have: $\ln \gamma_{2} \simeq \ln \frac{1}{\left|\mathbf{C}_{Q}\right|^{2}}+\frac{a_{21}}{L-2}+\frac{a_{22}}{(L-2)^{2}}+$ $\frac{1}{2}\left(\frac{a_{21}}{L-2}+\frac{a_{22}}{(L-2)^{2}}\right)^{2}$. As $\frac{1}{L-2} \simeq \frac{1}{L}\left(1+\frac{2}{L}+\frac{4}{L^{2}}\right)$ and $\frac{1}{(L-2)^{2}} \simeq \frac{1}{L^{2}}\left(1+\frac{4}{L}+\frac{12}{L^{2}}\right)$, we finally obtain, up to $1 / L^{2}: \ln \gamma_{2} \simeq \ln \frac{1}{\left|\mathbf{C}_{Q}\right|^{2}}+\frac{a_{21}}{L}+\frac{2 a_{21}+a_{22}+\frac{1}{2} a_{21}^{2}}{L^{2}}$. Therefore, after some algebra, we have,

$$
\begin{aligned}
& \ln \mu_{2, T \mid \mathcal{H}_{1}}-\ln \mu_{T \mid \mathcal{H}_{1}}^{2}=(B+C)-A+\ln \gamma_{2}-\ln \gamma_{1} \\
& \simeq \frac{\Delta}{L}+\frac{M^{2}+(2 M+3) \Delta+\frac{7}{4} \Delta^{2}+\left(a_{22}-2 a_{12}\right)}{L^{2}} .
\end{aligned}
$$


We obtain now the final large- $L$ results under $\mathcal{H}_{0}$ and $\mathcal{H}_{1}$ :

Hypothesis $\mathcal{H}_{0}:$ from (32) and (31): $\frac{1}{\beta_{0}} \simeq \frac{1}{2}\left(1+\frac{A}{C+D}\right)+1$. Thus,

$$
\frac{1}{\beta_{0}} \simeq \frac{3}{2}+\frac{\frac{M(M-1)}{L}\left(1+\frac{2 M+5}{6 L}\right)}{\frac{M(M-1)}{L^{2}}\left(1+\frac{2(M+4)}{3 L}\right)} \simeq L-\frac{M+1}{3} .
$$

From (33): $\alpha_{0} \simeq \frac{1}{2}\left(\frac{1}{\beta_{0}}-\frac{1}{2}\right) A$. Thus, using (90): $\alpha_{0} \simeq\left(L-\frac{2 M+5}{6}\right) \frac{M(M-1)}{2 L}\left(1+\frac{2 M+5}{6 L}\right)$, and, for large $L$,

$$
\alpha_{0} \simeq \frac{1}{2} M(M-1)
$$

Hypothesis $\mathcal{H}_{1}$ : using (32) and (31) under $\mathcal{H}_{1}$ with terms up to $\frac{1}{L}$, we have: $\frac{1}{\beta_{1}} \simeq \frac{\left(B+C+\ln \gamma_{2}\right) / 2}{-A+(B+C)-\ln \left(\gamma_{1} / \gamma_{2}\right)}+$ 1. After some algebra,

$$
\frac{1}{\beta_{1}} \simeq \frac{M(M-1)+2 \Delta+L \ln \frac{1}{\left|\mathbf{C}_{Q}\right|^{2}}}{2 \Delta}+1 .
$$

Using (33) under $\mathcal{H}_{1}$ with terms up to $\frac{1}{L}$,

$$
\alpha_{1} \simeq \frac{1}{2}\left(\frac{1}{\beta_{1}}-\frac{1}{2}\right)\left(\frac{M(M-1)+\Delta}{L}+\ln \frac{1}{\left|\mathbf{C}_{Q}\right|^{2}}\right) .
$$

\section{REFERENCES}

[1] T. Yucek and H. Arslan, "A survey of spectrum sensing algorithms for cognitive radio applications," IEEE Commun. Surv. Tutor., vol. 11, no. 1, pp. 116-130, 2009.

[2] E. Axell, G. Leus, E. Larsson and H. Poor, "Spectrum sensing for cognitive radio: State-of-the-art and recent advances," IEEE Signal Process. Mag., vol. 29, no. 3, pp. 101-116, May 2012.

[3] H. Urkowitz, "Energy detection of unknown deterministic signals," Proc. IEEE, vol. 55, no. 4, pp. 523-531, Apr. 1967.

[4] F. F. Digham, M. S. Alouini, and M. K. Simon, "On the energy detection of unknown signals over fading channels," IEEE Trans. Commun., vol. 55, no. 1, pp. 21-24, Jan. 2007.

[5] R. Tandra and A. Sahai, "Snr walls for signal detection," IEEE J. Sel. Topics Signal Process., vol. 2, no. 1, pp. 4-17, Feb. 2008.

[6] A. Taherpour, Y. Norouzi, M. Nasiri-Kenari, A. Jamshidi, and Z. Zeinalpour-Yazdi, "Asymptotically optimum detection of primary user in cognitive radio networks," IET Commun., vol. 1, pp. 1138-1145, Dec. 2007.

[7] A. Singh, M. R. Bhatnagar, and R. K. Mallik, "Optimization of cooperative spectrum sensing with an improved energy detector over imperfect reporting channels," in Proc. IEEE Veh. Tech. Conf. (VTC Fall), San Francisco, 2011, pp. 1-5.

[8] Q. Chen, M. Motani, W.-C. Wong, and A. Nallanathan, "Cooperative spectrum sensing strategies for cognitive radio mesh networks,” IEEE J. Sel. Topics Signal Process., vol. 5, no. 1, pp. 56-67, Feb. 2011.

[9] G. Taricco, "Optimization of linear cooperative spectrum sensing for cognitive radio networks," IEEE J. Sel. Topics Signal Process., vol. 5, no. 1, pp. 77-86, Feb. 2011. 
[10] Y. Zou, Y.-D. Yao, and B. Zheng, "A selective-relay based cooperative spectrum sensing scheme without dedicated reporting channels in cognitive radio networks," IEEE Trans. Wireless Commun., vol. 10, no. 4, pp. 1188-1198, Apr. 2011.

[11] Y. Zou, Y.-D. Yao, and B. Zheng, "Cooperative relay techniques for cognitive radio systems: spectrum sensing and secondary user transmissions," IEEE Commun. Mag., vol. 50, no. 4, pp. 98-103, Apr. 2012.

[12] L. Shen, H. Wang, W. Zhang, and Z. Zhao, "Multiple antennas assisted blind spectrum sensing in cognitive radio channels," IEEE Commun. Lett., vol. 16, no. 1, pp. 92-94, Jan. 2012.

[13] E. Axell and E. Larsson, "Optimal and sub-optimal spectrum sensing of ofdm signals in known and unknown noise variance," IEEE J. Sel. Areas Commun., vol. 29, no. 2, pp. 290-304, Feb. 2011.

[14] S. Sedighi, A. Taherpour, and J. Sala, "Spectrum Sensing Using Correlated Receiving Multiple Antennas in Cognitive Radios,” IEEE Trans. Wireless Commun., vol. 12, no. 11, pp. 5754-5766 , Nov. 2013.

[15] H. Nguyen, E. De Carvalho, and R. Prasad, "Spectrum sensing for cognitive radio based on multiple antennas," in Proc. IEEE Veh. Tech. Conf. (VTC Spring), Yokohama, 2012, pp. 1-5.

[16] A. Taherpour, M. Nasiri-Kenari, and S. Gazor, "Multiple antenna spectrum sensing in cognitive radios," IEEE Trans. Wireless Commun., vol. 9, no. 2, pp. 814-823, Feb. 2010.

[17] P. Bianchi, M. Debbah, M. Maïda, and J. Najim, "Performance of statistical tests for single-source detection using random matrix theory," IEEE Trans. Inf. Theory, vol. 57, no. 4, pp. 2400-2419, Apr. 2011.

[18] P. Wang, J. Fang, N. Han, and H. Li, "Multiantenna-assisted spectrum sensing for cognitive radio," IEEE Trans. Veh. Technol., vol. 59, no. 4, pp. 1791-1800, May 2010.

[19] S. Sedighi, A. Taherpour, and S. S.M. Monfared, "Bayesian generalised likelihood ratio test-based multiple antenna spectrum sensing for cognitive radios,” IET Commun., vol. 7, no. 18, pp. 2151-2165, Dec. 2013.

[20] R. Zhang, T. Lim, Y. Liang, and Y. Zeng, "Multi-antenna based spectrum sensing for cognitive radios: A glrt approach," IEEE Trans. Commun., vol. 58, no. 1, pp. 84-88, Jan. 2010.

[21] D. Ramírez, G. Vazquez-Vilar, R. López-Valcarce, J. Vía, and I. Santamaría, "Detection of rank-p signals in cognitive radio networks with uncalibrated multiple antennas," IEEE Trans. Signal Process., vol. 59, no. 8, pp. 3764-3774, Aug. 2011.

[22] R. López-Valcarce, G. Vazquez-Vilar, and J. Sala, "Multiantenna spectrum sensing for cognitive radio: overcoming noise uncertainty," in Proc. 2nd Int. Workshop on Cognitive Inform. Process. (CIP), Elba, 2010, pp. 310-315.

[23] A. Leshem and A. Van der Veen, "Multichannel detection and spatial signature estimation with uncalibrated receivers," in Proc. 11th IEEE Signal Process. Workshop on Statistical Signal Process., Singapore, 2001, pp. 190-193.

[24] A. Mariani, A. Giorgetti and M. Chiani, "Test of Independence for Cooperative Spectrum Sensing with Uncalibrated Receivers," Globecom 2012 . Cognitive Radio and Networks Symposium, 2012.

[25] J. K. Tugnait, "On Multiple Antenna Spectrum Sensing Under Noise Variance Uncertainty and Flat Fading," IEEE Transactions on Signal Processing, Vol. 60, No. 4, page(s): 1823 - 1832, April 2012.

[26] T. Weiss and F. Jondral, "Spectrum poolingAn innovative strategy for the enhancement of spectrum efficiency," IEEE Commun. Mag., vol. 42, no. 3, pp. S8-14, Mar. 2004.

[27] T. Weiss, J. Hillenbrand, A. Krohn, and F.K. Jondral, "Mutual interference in OFDM-based spectrum pooling systems," in Proc. IEEE Vehicular Technology Conf., Milan, 2004, pp. 1873-1877.

[28] H. Tang, "Some physical layer issues of wideband cognitive radio systems," in Proc. IEEE Int. Symp. New Frontiers Dynamic Spectrum Access Networks, Baltimore, 2005, pp. 151-159. 
[29] H. Neudecker and A. Wesselman, "The asymptotic variance matrix of the sample correlation matrix," Linear Algebra Applicat., vol. 127, pp. 589-599, 1990.

[30] M. Browne and A. Shapiro, "The asymptotic covariance matrix of sample correlation coefficients under general conditions," Linear Algebra Applicat., vol. 82, pp. 169-176, Oct. 1986.

[31] T. Kollo and K. Ruul, "Approximations to the distribution of the sample correlation matrix,” J. Multivar. Anal., vol. 85, no. 2, pp. 318-334, May 2003.

[32] G. Vazquez-Vilar, R. L'pez-Valcarce, and J. Sala, "Multiantenna spectrum sensing exploiting spectral a priori information," IEEE Trans. Wireless Commun., vol. 23, no. 2, pp. 4345-4355, Dec. 2005.

[33] J. Sala, G. Vazquez-Vilar, and R. Lopez-Valcarce, "Multiantenna GLR detection of rank-one signals with known power spectrum in white noise with unknown spatial correlation,” IEEE Trans. Signal Process., vol. 60, no. 6, pp. 3065-3078, Jun. 2012.

[34] D. Ramírez, J. Vía, I. Santamaría, and L. L. Scharf, "Locally Most Powerful Invariant Tests for Correlation and Sphericity of Gaussian Vectors," IEEE Trans. Inf. Theory, vol. 59, no. 4, pp. 2128-2141, Apr. 2013.

[35] H. H. Andersen, M. Hjbjerre, D. Srensen, and P. S. Eriksen, Linear and Graphical Models for the Multivariate Complex Normal Distribution, vol. 101. New York: Springer-Verlag, 1995.

[36] I. Ryzhik, A. Jeffrey, and D. Zwillinger, Table of Integrals, Series and Products. Waltham, Massachusetts: Academic Press, 2007.

[37] Y. Zeng and Y.-C. Liang, "Maximum-minimum eigenvalue detection for cognitive radio," in Proc. IEEE Int. Symp. Personal, Indoor and Mobile Radio Commun. (PIMRC), Athens, 2007, pp. 1-5.

[38] M. Ghosh, "Basus theorem with applications: a personalistic review," Sankhyā: The Indian J. Stat., Ser. A, vol. 64, no. 3, pp. 509-531, Oct. 2002.

[39] R. Couillet and M. Debbah, Random Matrix Methods for Wireless Communications. Cambridge, England: Cambridge University Press, 2011.

[40] N. Dunford and J. T. Schwartz, Linear Operators, Part II: Spectral Theory, Self Adjoint Operators in Hilbert Space. Hoboken, New Jersey: John Wiley and Sons Inc., 1963.

[41] E.T. Bell, "Exponential polynomials," Ann. Math., vol. 35, no. 2, pp. 258-277, Apr. 1934.

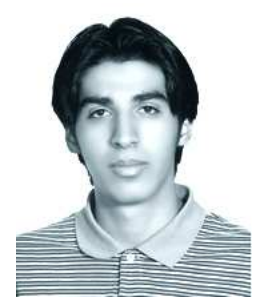

Saeid Sedighi was born in Tehran, Iran, in 1987. He received the B.Sc degree in Electrical Engineering from the Islamic Azad University, Tehran, Iran, in 2010 and the M.Sc. degree in Telecommunications Engineering from Imam Khomeini International University (IKIU), Qazvin, Iran, in 2012. Since Otober 2012, he has been serving as a research assistant in the Signal Processing and Information Theory (SPIT) lab at the Electrical Engineering Department, Imam Khomeini International University (IKIU). He has been involved in a research project at Qatar University (QU) from September 2014 until April 2015.

He has His research interests are cognitive radio, statistical signal processing, detection and estimation theory, and cooperative communications. 


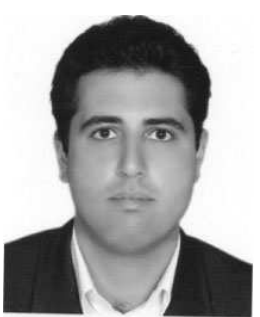

Abbas Taherpour (S07-M09) received the B.Sc. and PhD degrees in Electrical Engineering, Communication Systems, from Sharif University of Technology, Tehran, Iran in 2002 and 2009, respectively. He also received his M.Sc. degree from Tehran University, Tehran, Iran in Electrical Engineering, Communication Systems in 2004. He is currently an assistant professor with the Electrical Engineering Department of Imam Khomeini International University (IKIU), Qazvin, Iran. He has been a visiting scholar at Queen's University, ON, Canada form June 2007 until June 2008 and has participated in several wireless communications and signal processing projects at the regional and European level with industry and institutions. His research interests are cognitive radio, statistical signal processing and wireless communications.

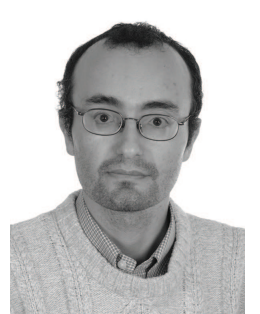

Josep Sala-Alvarez (M'86) born in Barcelona, Spain, in 1967. He received the M.Sc. and Ph.D. degrees in telecommunications engineering from the Technical University of Catalonia (UPC), Barcelona, Spain, in 1991 and 1995, respectively. During 1992, he worked at the European Space Operations Centre (ESOC) of the European Space Agency (ESA), Darmstadt, Germany, in the area of software engineering for telemetry processing. From 1993 to late 1994, he held a grant from the Generalitat de Catalunya in support of the Ph.D. degree at the Department of Signal Theory and Communications, UPC. In 1994, he joined this department as Assistant Professor and was promoted to Associate Professor in 1997. He has participated in spacerelated communication projects for ESA and in wireless communications projects at the national/European level with industry and institutions. His current research interests are in the field of signal processing, communications, and information theory. Dr. Sala is recipient of the IEEE Signal Processing Society Best (Senior) PaperAward 2003, the International Symposium on Turbo-Codes and Applications (ISTC03) Best Poster Paper Award, and the Best Ph.D. Thesis in Telecommunications National Award (Spain, 1995). Dr. Sala is an IEEE Senior Member. 


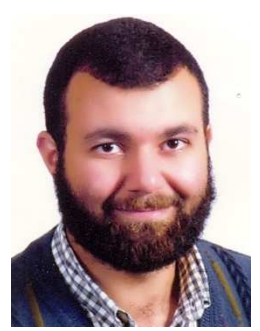

Tamer Khattab (M’ 94) received his Ph.D. in Electrical and Computer Engineering from the University of British Columbia (UBC), Vancouver, BC, Canada in 2007, M.Sc. in Electronics and Communications Engineering and B.Sc. in Electronics and Communications Engineering from Cairo University, Giza, Egypt. Dr. Khattab has been an assistant professor of Electrical Engineering at Qatar University (QU) since 2007. He is also a senior member of the technical staff at Qatar Mobility Innovation Center (QMIC), an R\&D center owned by QU and funded by Qatar Science and Technology Park (QSTP). Between 2006 and 2007 he was a postdoctoral fellow at the University of British Columbia working on prototyping advanced Gigabit/sec wireless LAN baseband transceivers. During 2000-2003 Dr. Khattab joined Alcatel Canada's Network and Service Management R\&D in Vancouver, BC, Canada as a member of the technical staff working on development of core components for Alcatel 5620 network and service manager. Between 1994 and 1999 he was with IBM wtc. Egypt as a software development team lead working on development of several client-server corporate tools for IBM labs. Dr. Khattab's research interests cover physical layer transmission techniques in optical and wireless networks, information theoretic aspects of communication systems and MAC layer protocol design and analysis. 